\title{
DISTRIBUTED CONTROL OF SPATIALLY REVERSIBLE INTERCONNECTED SYSTEMS WITH BOUNDARY CONDITIONS*
}

\author{
CÉDRIC LANGBORT ${ }^{\dagger}$ AND RAFFAELLO D’ANDREA ${ }^{\ddagger}$
}

\begin{abstract}
We present a class of spatially interconnected systems with boundary conditions that have close links with their spatially invariant extensions. In particular, well-posedness, stability, and performance of the extension imply the same characteristics for the actual, finite extent system. In turn, existing synthesis methods for control of spatially invariant systems can be extended to this class. The relation between the two kinds of systems is proved using ideas based on the "method of images" of partial differential equations theory and uses symmetry properties of the interconnection as a key tool.
\end{abstract}

Key words. spatially distributed control, symmetric systems, boundary conditions

AMS subject classifications. 93A14, 93A15, 93B36

DOI. $10.1137 / \mathrm{S} 0363012902415803$

1. Introduction. Many systems consist of the interconnection of a large number of identical subunits which interact with their nearest neighbors. Examples of such interconnected systems include formations of autonomous vehicles [11], [22], crossdirectional control in the pulp and paper and chemical process industry [13], [14], "smart structures" (large arrays of distributed micro electromechanical actuators and sensors) [2], and semidiscretized partial differential equations [3].

Over the years, several frameworks for control of interconnected systems have been proposed that all assumed the existence of a particular mathematical structure induced by the interconnection. Early works [3], [17] showed that some systems, especially semidiscretized partial differential equations, can sometimes be treated as systems over modules. More recently, the papers [1], [5], [15] have considered so-called spatially invariant systems and used Fourier techniques or algebraic transformations to derive implementable and scalable optimal control algorithms, even in the limit of an infinite number of subunits. While some practical systems can be accurately modelled as being spatially invariant (e.g., circular plastic extrusion machines or very large arrays of sensors and actuators), most examples do not fall into this category because they are of finite extent and possess boundary conditions. This is why much of the current research is geared toward spatially varying systems, in an effort to adapt methods from the monodimensional time-varying case [8].

The approach taken in this paper is different, as we show that analysis and synthesis for the actual finite extent system with boundary conditions can sometimes be performed by studying a larger, spatially invariant system. The key assumption for this result is another structural property which we call spatial reversibility. In short,

*Received by the editors October 5, 2002; accepted for publication (in revised form) August 23, 2004; published electronically June 14, 2005. This work was supported in part by a National Science Foundation CAREER Award for "Robust and Optimal Control of Interconnected Systems" and in part by the Air Force Office of Scientific Research under grant F49620-01-1-0119.

http://www.siam.org/journals/sicon/44-1/41580.html

${ }^{\dagger}$ Center for the Mathematics of Information, California Institute of Technology, 1200 E. California Blvd., MS 136-93, Pasadena, CA 91125 (clangbort@ist.caltech.edu). This work was performed while the author was affiliated with T\&AM, Cornell University.

${ }_{\ddagger}^{\ddagger}$ Mechanical and Aerospace Engineering, Cornell University, 101 Rhodes Hall, Ithaca, NY 14853 (rd28@cornell.edu). 
we prove that a lack of spatial invariance can be made up for by spatial reversibility of the finite extent system with boundary conditions and that, in turn, any technique designed for spatially invariant systems can be used in that case too. A major difference between spatial invariance and spatial reversibility is that the former is a property of the interconnection while the latter is a property of the subsystems. In the language of [6], spatial reversibility is an internal symmetry while spatial invariance is a global symmetry of the system.

Our method borrows concepts from two different lines of thought. First, the idea of associating a larger spatially invariant system to the actual finite extent system is very similar in nature to the "lifting technique" introduced in [12] to relate linear time-periodic to linear time-invariant systems or to the method of [13] used to prove robustness of cross-directional controllers. Second, the motivation for considering symmetries of the system comes from the so-called "method of images" used in potential theory. The main issue that has to be addressed in order to establish a real link between finite extent and spatially invariant systems is that the boundary conditions are lost in this correspondence. One would like to consider finite extent systems, the solution of which can be recovered from the spatially invariant system in spite of this information loss. The method of images gives an example in which such a situation is at hand, although in a different context. It essentially states that boundary conditions for Laplace's equation

$$
\Delta u=0 \text { in } U ; \quad u=g \text { on } \partial U
$$

on some simple domains $U \subset \mathbb{R}^{n}$ (e.g., half-spaces) can be dropped as such since its solution can be determined by solving a similar equation on the whole of $\mathbb{R}^{n}$, provided "mirror-image singularities" are introduced [20], [9]. The main reason why this technique works is that the Laplacian has some symmetry properties-namely, it commutes with any isometry of $\mathbb{R}^{n}$. It is thus natural to hope that spatial symmetry is also relevant for our problem.

It should be noted that an "embedding technique" similar to the method of images was already used in [3] to handle boundary conditions for the particular example of the semidiscretized heat equation on a finite interval. However, it was not emphasized that the possibility of using such a technique was due to symmetries of the problem.

The paper is organized as follows. After giving general preliminaries and notions on finite extent and spatially invariant systems in sections 2 and 3, we define spatial reversibility in section 4 and explain how it allows us to relate well-posedness, stability, and performance of these two kinds of systems. Section 5 presents practical examples of spatially reversible systems and section 6 is devoted to synthesis of distributed controllers for an $\mathcal{H}_{\infty}$ criterion. In particular, section 6.2 is largely independent from the rest, as it illustrates how the specific results of [5] can be adapted to this finite extent problem. Finally section 7 contains some generalizations of the core results, while concluding remarks can be found in section 8 .

2. Modelling spatially interconnected systems. In this section, we introduce our notation and define the basic objects of interest. The goal is to provide a framework in which infinite, periodic, and finite extent systems can be handled simultaneously.

2.1. Signal spaces. Unless otherwise stated, $\mathbb{M}$ will stand for any one of the following three sets: $\{1, \ldots, L\}$ for some integer $L>0, \mathbb{Z}_{2 L}$ (the group of integers 
modulo $2 L$ ), and $\mathbb{Z}$. We define $\ell_{2}^{q}(\mathbb{M})$ as the space of functions $x: \mathbb{M} \rightarrow \mathbb{R}^{q}$ such that

$$
\|x\|_{\ell_{2}^{q}(\mathbb{M})}^{2}:=\sum_{s \in \mathbb{M}} x(s)^{*} x(s)<\infty .
$$

Then $\mathcal{L}_{2}^{q}(\mathbb{M})$ is defined as the Hilbert space of functions $x: \mathbb{R}^{+} \rightarrow \ell_{2}^{q}(\mathbb{M})$ such that

$$
\|x\|_{\mathcal{L}_{2}^{q}(\mathbb{M})}^{2}:=\int_{0}^{\infty}\|x(t)\|_{\ell_{2}^{q}(\mathbb{M})}^{2} d t<\infty
$$

When the dimension of the target space is clear from the context or is irrelevant, we omit the superscript $q$ and simply write $\ell_{2}(\mathbb{M})$ and $\mathcal{L}_{2}(\mathbb{M})$. Finally, if $J$ is a matrix, we will abuse notation and identify it with the operator that associates $y \in \ell_{2}(\mathbb{M})$ to $x \in \ell_{2}(\mathbb{M})$ such that $y(s)=J x(s)$ for all $s \in \mathbb{M}$.

2.2. Systems. Let a linear time-invariant, finite dimensional, dynamical system with input $\left(d, v^{+}, v^{-}\right)$and output $\left(z, w^{+}, w^{-}\right)$be given in state space by

$$
\begin{aligned}
\frac{d}{d t} x(t) & =A_{\mathrm{TT}_{\mathrm{T}}} x(t)+A_{\mathrm{TS}_{+}} v^{+}(t)+A_{\mathrm{TS}_{-}} v^{-}(t)+B_{\mathrm{T}} d(t) ; x(0)=x^{0}, \\
w^{+}(t) & =A_{\mathrm{ST}_{+}} x(t)+A_{\mathrm{SS}_{+,+}} v^{+}(t)+A_{\mathrm{SS}_{+,-}} v^{-}(t)+B_{\mathrm{S}_{+}} d(t), \\
w^{-}(t) & =A_{\mathrm{ST}_{-}} x(t)+A_{\mathrm{SS}_{-},+} v^{+}(t)+A_{\mathrm{SS}_{-,-}} v^{-}(t)+B_{\mathrm{S}_{-}} d(t), \\
z(t) & =C_{\mathrm{T}} x(t)+C_{\mathrm{S}_{+}} v^{+}(t)+C_{\mathrm{S}_{-}} v^{-}(t)+D d(t),
\end{aligned}
$$

where $x(t), d(t)$, and $z(t)$ belong to $\mathbb{R}^{n_{\mathrm{T}}}, \mathbb{R}^{m}$, and $\mathbb{R}^{p}$, respectively, for all $t \geq 0$, and $v^{+}(t), v^{-}(t), w^{+}(t), w^{-}(t)$ all belong to $\mathbb{R}^{n}$. We also let $n_{\mathrm{S}}:=2 n$. To such a system, which we call the basic building block, we can associate three different spatially interconnected systems as follows.

2.2.1. Infinite system. Let the shift operator $\mathbf{S}$ be defined on $\ell_{2}(\mathbb{Z})$ by

$$
(\mathbf{S} v)(s):=v(s+1) \text { for all } s .
$$

$\mathbf{S}$ is clearly an isometry. We introduce the operator $\Delta_{\mathrm{S}}:=\operatorname{diag}\left(\mathbf{S} I_{n}, \mathbf{S}^{-1} I_{n}\right)$ on $\ell_{2}^{n_{\mathrm{S}}}(\mathbb{Z})$. The infinite system associated to building block (1) is described by

$$
\begin{aligned}
\frac{d}{d t} x(t) & =A_{\mathrm{TT}} x(t)+A_{\mathrm{TS}} v(t)+B_{\mathrm{T}} d(t) \quad \text { for all } t \geq 0 ; x(0)=x^{0}, \\
\left(\Delta_{\mathrm{S}}-A_{\mathrm{SS}}\right)[v(t)] & =A_{\mathrm{ST}} x(t)+B_{\mathrm{S}} d(t) \text { for all } t \geq 0, \\
z(t) & =C_{\mathrm{T}} x(t)+C_{\mathrm{S}} v(t)+D d(t) \quad \text { for all } t \geq 0,
\end{aligned}
$$

where we have used the shorthand

$$
\begin{gathered}
A_{\mathrm{ST}}:=\left(\begin{array}{c}
A_{\mathrm{ST}_{+}} \\
A_{\mathrm{ST}_{-}}
\end{array}\right), A_{\mathrm{SS}}:=\left(\begin{array}{cc}
A_{\mathrm{SS}_{+}++} & A_{\mathrm{SS}_{+,-}} \\
A_{\mathrm{SS}_{-},+} & A_{\mathrm{SS}_{-,-}}
\end{array}\right), B_{\mathrm{S}}:=\left(\begin{array}{c}
B_{\mathrm{S}_{+}} \\
B_{\mathrm{S}_{-}}
\end{array}\right), \\
A_{\mathrm{TS}}:=\left(\begin{array}{ll}
A_{\mathrm{TS}_{+}} & A_{\mathrm{TS}_{-}}
\end{array}\right), C_{\mathrm{S}}:=\left(\begin{array}{ll}
C_{\mathrm{S}_{+}} & C_{\mathrm{S}_{-}}
\end{array}\right) .
\end{gathered}
$$

In (3), the triple $(x(t), v(t), z(t))$ is sought in $\ell_{2}^{n_{\mathrm{T}}}(\mathbb{Z}) \times \ell_{2}^{n_{\mathrm{s}}}(\mathbb{Z}) \times \ell_{2}^{p}(\mathbb{Z})$ for all $t \geq 0$, when an initial condition $x^{0} \in \ell_{2}^{n_{\mathrm{T}}}(\mathbb{Z})$ and a disturbance $d$ such that $d(t) \in \ell_{2}^{m}(\mathbb{Z})$ for all $t \geq 0$ are given. The question of whether such a triple exists is addressed in section 3 . We answer it by rewriting the infinite set of differential-algebraic equations 


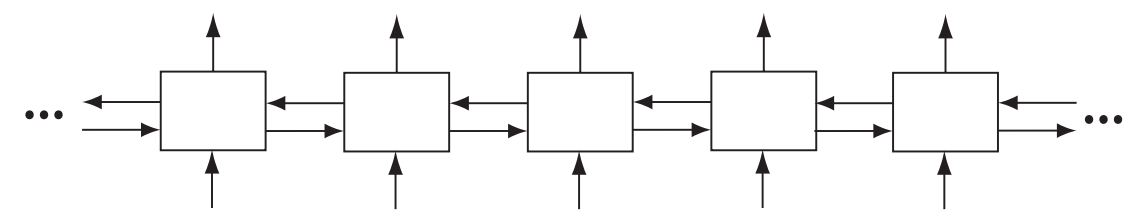

(a)

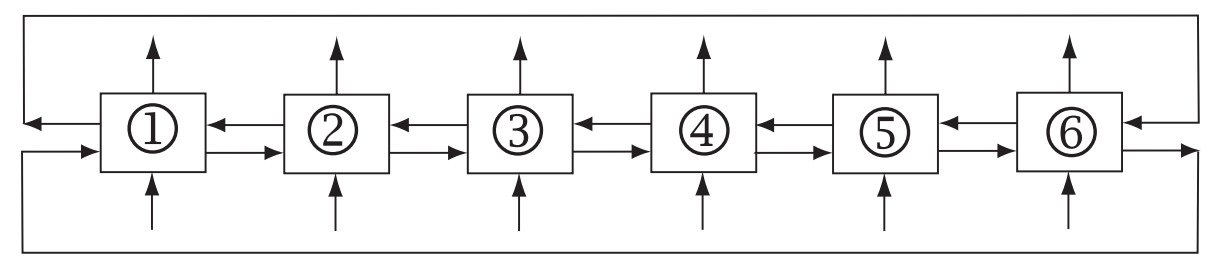

(b)

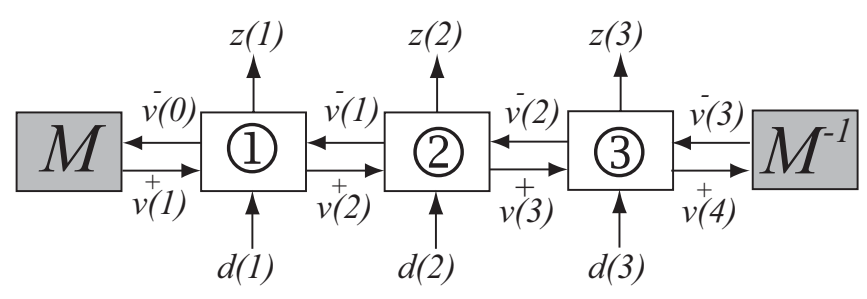

(c)

FIG. 1. The three types of interconnected systems. (a) Infinite system. (b) Periodic system for $L=3$. (c) Finite extent system for $L=3$ (time dependence of signals is not indicated to simplify notation).

(3) as an abstract differential equation on the Hilbert space $\ell_{2}^{n_{\mathrm{T}}}(\mathbb{Z})$. However, it should be noted that infinite interconnected systems appear more naturally in the form of (3) than as abstract differential equations. Indeed, consider the block diagram pictured in Figure 1(a). Each box stands for an instance of the basic building block (1) that exchanges signals $v^{+}, v^{-}, w^{+}$, and $w^{-}$with its neighbors according to the interconnection relation

$$
\begin{aligned}
& {\left[v^{+}(t)\right](s+1)=\left[w^{+}(t)\right](s), s \in \mathbb{Z},} \\
& {\left[v^{-}(t)\right](s-1)=\left[w^{-}(t)\right](s), s \in \mathbb{Z},}
\end{aligned}
$$

where we have indexed the subsystems by $s \in \mathbb{Z}$ and considered all signals mentioned before as vector-valued functions on $\mathbb{Z}$ for all $t$. Introducing $v$ such that

$$
[v(t)](s):=\left(\left[v^{+}(t)\right](s),\left[v^{-}(t)\right](s)\right) \text { for all } t \geq 0
$$

and recalling the definition of the operator $\Delta_{\mathrm{S}}$, it is easy to see that conditions (4) and the state space description of each subsystem yield differential-algebraic equations (3).

2.2.2. Periodic system. A periodic interconnected system is also captured by (3) but with the operators $\mathbf{S}$ and $\Delta_{\mathrm{S}}$ now defined, respectively, on $\ell_{2}\left(\mathbb{Z}_{2 L}\right)$ and 
$\ell_{2}^{n_{\mathrm{S}}}\left(\mathbb{Z}_{2 L}\right)$. In particular, the shift operator $\mathbf{S}$ is still defined by (2) but addition should now be understood modulo $2 L$. Accordingly, the triple $(x(t), v(t), z(t))$ is sought in $\ell_{2}^{n_{\mathrm{T}}}\left(\mathbb{Z}_{2 L}\right) \times \ell_{2}^{n_{\mathrm{S}}}\left(\mathbb{Z}_{2 L}\right) \times \ell_{2}^{p}\left(\mathbb{Z}_{2 L}\right)$ for all $t \geq 0$, when an initial condition $x^{0} \in \ell_{2}^{n_{\mathrm{T}}}\left(\mathbb{Z}_{2 L}\right)$ and a disturbance $d$ such that $d(t) \in \ell_{2}^{m}\left(\mathbb{Z}_{2 L}\right)$ for all $t \geq 0$ are given.

The physical interconnection corresponding to a periodic system is illustrated in Figure 1(b). The subsystems are again instances of the basic building block and are interconnected according to the relation

$$
\begin{aligned}
& {\left[v^{+}(t)\right](s+1)=\left[w^{+}(t)\right](s), s \in \mathbb{Z}_{2 L},} \\
& {\left[v^{-}(t)\right](s-1)=\left[w^{-}(t)\right](s), s \in \mathbb{Z}_{2 L} .}
\end{aligned}
$$

For reasons that should become clear in section 3 , we will say that periodic and infinite systems are spatially invariant.

2.2.3. Finite extent system. Unlike infinite and periodic systems that can be readily defined in a formal setting, finite extent systems are easier to introduce through the physical interconnection they describe. Consider the block diagram of Figure 1(c). As in the infinite and periodic case, each box represents an instance of the basic building block, except the two end ones, which specify boundary conditions. More precisely, if we index subsystems by $1 \leq s \leq L$, the interconnection relation between neighboring subsystems now is

$$
\begin{aligned}
{\left[v^{+}(t)\right](s+1) } & =\left[w^{+}(t)\right](s), 1 \leq s \leq L-1, \\
{\left[v^{-}(t)\right](s-1) } & =\left[w^{-}(t)\right](s), 2 \leq s \leq L, \\
{\left[v^{+}(t)\right](1) } & =M\left[w^{-}(t)\right](1), \\
{\left[v^{-}(t)\right](L) } & =M^{-1}\left[w^{+}(t)\right](L),
\end{aligned}
$$

where $M$ is a nonsingular matrix called the boundary conditions matrix. We can represent such a finite extent system by a set of differential-algebraic equations formally similar to that describing infinite and periodic systems. To this end, we need to introduce the operator $\Delta_{\mathrm{BC}}$ as follows. First, if $v=\left(v^{+}, v^{-}\right)$belongs to $\ell_{2}^{n_{\mathrm{S}}}(\{1, \ldots, L\})$, we define the vector $\vec{v} \in \mathbb{R}^{n_{\mathrm{S}} L}$ by

$$
\vec{v}=\left(v^{+}(1), \ldots, v^{+}(L), v^{-}(1), \ldots, v^{-}(L)\right) .
$$

The map $\rightarrow$ is an isomorphism of $\mathbb{R}$-vector spaces and its inverse will be denoted $\leftarrow$. As a consequence, we can define another isomorphism, also denoted $\leftarrow$, between the space of $n_{\mathrm{S}} L \times n_{\mathrm{S}} L$ real matrices and the space of endomorphisms of $\ell_{2}^{n_{\mathrm{S}}}(\{1, \ldots, L\})$ by

$$
\overleftarrow{J} v:=\overleftarrow{(J \vec{v})} \text { for all } J \in \mathbb{R}^{n_{\mathrm{S}} L \times n_{\mathrm{s}} L}, v \in \ell_{2}^{n_{\mathrm{s}}}(\{1, \ldots, L\})
$$

With this notation, we can rewrite the interconnection relation (7) as

$$
w=\overleftarrow{\mathcal{C}} v
$$

for the invertible interconnection matrix $\mathcal{C}$. In the remainder of this paper, we will let $\overleftarrow{\mathcal{C}}=: \Delta_{\mathrm{BC}}$. Then, introducing again signal $v$ as per (5), a finite extent system can be represented by the following set of differential-algebraic equations:

$$
\begin{aligned}
\frac{d}{d t} x(t) & =A_{\mathrm{TT}} x(t)+A_{\mathrm{TS}} v(t)+B_{\mathrm{T}} d(t) \text { for all } t \geq 0 ; x(0)=x^{0}, \\
\left(\Delta_{\mathrm{BC}}-A_{\mathrm{SS}}\right)[v(t)] & =A_{\mathrm{ST}} x(t)+B_{\mathrm{S}} d(t) \text { for all } t \geq 0, \\
z(t) & =C_{\mathrm{T}} x(t)+C_{\mathrm{S}} v(t)+D d(t) \text { for all } t \geq 0,
\end{aligned}
$$


which is formally similar to (3). The triple $(x(t), v(t), z(t))$ is sought in $\ell_{2}^{n_{\mathrm{T}}}(\{1, \ldots, L\}) \times$ $\ell_{2}^{n_{\mathrm{S}}}(\{1, \ldots, L\}) \times \ell_{2}^{p}(\{1, \ldots, L\})$ for all $t \geq 0$, when an initial condition $x^{0} \in \ell_{2}^{n_{\mathrm{T}}}(\{1, \ldots, L\})$ and a disturbance $d$ such that $d(t) \in \ell_{2}^{m}(\{1, \ldots, L\})$ for all $t \geq 0$ are given.

As already mentioned in the introduction, analysis is much more tractable for spatially invariant systems than for finite extent systems, especially if $L$ is large. Hence, it would be desirable to know what relationships exist between them. The main goal of the next sections is to show that stability and performance of the spatially invariant systems actually imply similar properties for the corresponding finite extent system, provided some reversibility properties are satisfied. A proof of this statement, which we call the method of images, as well as a precise definition of what we call "reversibility" are given in section 4. Before presenting these results, more should be said about well-posedness, stability, and performance.

3. Well-posedness, stability, and performance. We have just seen that all interconnected systems of interest can be captured by the following equations:

$$
\begin{aligned}
\frac{d}{d t} x(t) & =A_{\mathrm{TT}} x(t)+A_{\mathrm{TS}} v(t)+B_{\mathrm{T}} d(t) \quad \text { for all } t \geq 0 ; x(0)=x^{0}, \\
\left(\Delta-A_{\mathrm{SS}}\right)[v(t)] & =A_{\mathrm{ST}} x(t)+B_{\mathrm{S}} d(t) \text { for all } t \geq 0, \\
z(t) & =C_{\mathrm{T}} x(t)+C_{\mathrm{S}} v(t)+D d(t) \quad \text { for all } t \geq 0,
\end{aligned}
$$

where

$$
\begin{array}{cl}
\Delta=\Delta_{\mathrm{BC}} & \text { for a finite extent system, } \\
\Delta=\Delta_{\mathrm{S}} & \text { for an infinite or periodic system. }
\end{array}
$$

System (9) is said to be well-posed if the bounded linear operator $\left(\Delta-A_{\mathrm{SS}}\right): \ell_{2}^{n_{\mathrm{S}}}(\mathbb{M}) \rightarrow$ $\ell_{2}^{n_{s}}(\mathbb{M})$ is invertible. Assume system (9) is well-posed and let an initial state $x^{0} \in$ $\ell_{2}(\mathbb{M})$ and a disturbance $d \in \mathcal{L}_{2}(\mathbb{M})$ be given. We can write

$$
v(t)=\left(\Delta-A_{\mathrm{SS}}\right)^{-1}\left(A_{\mathrm{ST}} x(t)+B_{\mathrm{S}} d(t)\right) \text { for all } t \geq 0
$$

and, in turn, $x$ will satisfy

$$
\begin{aligned}
\frac{d}{d t} x(t) & =\mathbf{A} x(t)+\mathbf{B} d(t) \text { for all } t \geq 0, \\
x(0) & =x^{0},
\end{aligned}
$$

where

$$
\mathbf{A}=\left(A_{\mathrm{TT}}+A_{\mathrm{TS}}\left(\Delta-A_{\mathrm{SS}}\right)^{-1} A_{\mathrm{ST}}\right) ; \mathbf{B}=\left(B_{\mathrm{T}}+A_{\mathrm{TS}}\left(\Delta-A_{\mathrm{SS}}\right)^{-1} B_{\mathrm{S}}\right) .
$$

Note that operators $\mathbf{A}$ and $\mathbf{B}$ are bounded and thus, in particular, $\mathbf{A}$ generates a strongly continuous semigroup of operators $\{\Phi(t)\}_{t \geq 0}$ on $\ell_{2}(\mathbb{M})$. We can even write

$$
\Phi(t)=e^{t \mathbf{A}},
$$

where the exponential is defined by the usual power series. As a result [4], (11a) has a unique weak solution on $[0, T]$ for any $x^{0} \in \ell_{2}(\mathbb{M})$ and $d \in \mathcal{L}_{2}(\mathbb{M})$, which is the mild solution given by

$$
x(t)=e^{t \mathbf{A}} x^{0}+\int_{0}^{t} e^{(t-\tau) \mathbf{A}}(\mathbf{B} d(\tau)) d \tau .
$$


The solution of well-posed system (9) on the interval $[0, T]$ is the unique triple $(x, v, z)$ such that $x$ is the mild solution of (11a) on $[0, T]$ and (9c) and (10) are satisfied for all $0 \leq t \leq T$. It is not hard to see that if $(x, v, z)$ is the solution of a periodic or infinite system for initial condition $x^{0}$ and disturbance $d(t)$, then $(\tilde{x}, \tilde{v}, \tilde{z})$ is another solution for initial condition $\mathbf{S} x^{0}$ and disturbance $\mathbf{S} d(t)$, where

$$
\tilde{x}(t)=\mathbf{S}[x(t)], \tilde{v}(t)=\mathbf{S}[v(t)], \tilde{z}(t)=\mathbf{S}[z(t)] \text { for all } \mathrm{t} .
$$

This is the reason why we chose to call these systems spatially invariant. The physical explanation of this invariance is that all subsystems in block diagrams 1(a) and 1(b) are identical and interconnected to their neighbors in the same way.

We now show that well-posedness can be characterized algebraically.

Proposition 3.1. (i) A finite extent system is well-posed if and only if $(I-N)$ is invertible, where $N$ is defined as

$$
\left(\begin{array}{ccc|cc}
M A_{\mathrm{SS}_{-,+}} & & 0^{n \times n(L-1)} & M A_{\mathrm{SS}_{-,-}} & 0^{n \times n(L-1)} \\
\hline & \mathcal{T}_{\mathrm{L}}\left(A_{\mathrm{SS}_{+,+}}\right) & & \multicolumn{2}{|c}{\mathcal{T}_{\mathrm{L}}\left(A_{\mathrm{SS}_{+},-}\right)} \\
\hline \mathcal{T}_{\mathrm{U}}\left(A_{\mathrm{SS}_{-,+}}\right) & & \multicolumn{2}{|c}{\mathcal{T}_{\mathrm{U}}\left(A_{\mathrm{SS}_{-,-}}\right)} & M^{-1} A_{\mathrm{SS}_{+,-}}
\end{array}\right),
$$

and the rectangular Toeplitz matrices $\mathcal{T}_{\mathrm{U}}(K)$ and $\mathcal{T}_{\mathrm{L}}(K)$ in $\mathbb{R}^{n(L-1) \times n L}$ satisfy

$$
\mathcal{T}_{\mathrm{U}}(K):=\left(\begin{array}{ccccc}
0 & K & \cdots & \cdots & 0 \\
0 & \ddots & \ddots & & \vdots \\
\vdots & \ddots & \ddots & \ddots & \vdots \\
0 & \cdots & 0 & 0 & K
\end{array}\right) ; \mathcal{T}_{\mathrm{L}}(K):=\left(\begin{array}{ccccc}
K & 0 & \cdots & \cdots & 0 \\
0 & \ddots & \ddots & & \vdots \\
\vdots & \ddots & \ddots & \ddots & \vdots \\
0 & \cdots & 0 & K & 0
\end{array}\right)
$$

for any given $K \in \mathbb{R}^{n \times n}$.

(ii) A periodic (respectively, infinite) system is well-posed if and only if $(\Delta(\lambda)-$ $\left.A_{\mathrm{SS}}\right)$ is invertible for all $\lambda \in \mathbb{U}$ (respectively, for all $\lambda \in \partial \mathbb{D}$ ), where

$$
\Delta(\lambda):=\operatorname{diag}\left(\lambda I_{n}, \lambda^{-1} I_{n}\right) \text { and } \partial \mathbb{D}:=\{z \in \mathbb{C},|z|=1\}, \mathbb{U}:=\left\{z \in \mathbb{C}, z^{2 L}=1\right\} .
$$

Proof. (i) Since the interconnection matrix $\mathcal{C}$ is invertible, so is $\Delta_{\mathrm{BC}}$. It is easy to see that $N$ satisfies $\overleftarrow{N}=\Delta_{\mathrm{BC}}^{-1} A_{\mathrm{SS}}$. Hence matrix $(I-N)$ is nonsingular if and only if $\left(\Delta_{\mathrm{BC}}-A_{\mathrm{SS}}\right)$ is. Since $\ell_{2}(\{1, \ldots, L\})$ is finite dimensional, $\left(\Delta_{\mathrm{BC}}-A_{\mathrm{SS}}\right)^{-1}$ is bounded whenever it exists.

(ii) We first study the periodic case. Let $v \in \ell_{2}\left(\mathbb{Z}_{2 L}\right)$ be given. It has a discrete Fourier transform $\hat{v} \in \ell_{2}(\mathbb{U})$ defined by

$$
\hat{v}(\lambda)=\sum_{s=1}^{2 L} v(s) \lambda^{s} \text { for all } \lambda \in \mathbb{U} .
$$

Now assume $\left(\Delta(\lambda)-A_{\mathrm{SS}}\right)$ is invertible for all $\lambda \in \mathbb{U}$. Then $n$ defined by

$$
n(s)=\frac{1}{2 L} \sum_{\lambda \in \mathbb{U}} \lambda^{-s}\left(\Delta(\lambda)-A_{\mathrm{SS}}\right)^{-1} \hat{v}(\lambda)
$$

is a well-defined function on $\mathbb{Z}_{2 L}$. Also, noting that

$$
\hat{n}(\lambda)=\left(\Delta(\lambda)-A_{\mathrm{SS}}\right)^{-1} \hat{v}(\lambda) \text { for all } \lambda
$$


and using Parseval's identity, we get that

$$
\|n\|_{\ell_{2}\left(\mathbb{Z}_{2 L}\right)} \leq \max _{\lambda \in \mathbb{U}} \bar{\sigma}\left(\left(\Delta(\lambda)-A_{\mathrm{SS}}\right)^{-1}\right)\|v\|_{\ell_{2}\left(\mathbb{Z}_{2 L}\right)}<\infty .
$$

Finally, it is easy to check that it satisfies

$$
\left(\Delta_{\mathrm{S}}-A_{\mathrm{SS}}\right) n=v .
$$

This is the unique solution in $\ell_{2}\left(\mathbb{Z}_{2 L}\right)$ since any such solution must satisfy (15) and elements of $\ell_{2}\left(\mathbb{Z}_{2 L}\right)$ are fully specified by their Fourier coefficients. Hence, $\left(\Delta_{\mathrm{S}}-A_{\mathrm{SS}}\right)^{-1}$ is well defined on $\ell_{2}\left(\mathbb{Z}_{2 L}\right)$ and has norm less than $\max _{\lambda \in \mathbb{U}} \bar{\sigma}\left(\left(\Delta(\lambda)-A_{\mathrm{SS}}\right)^{-1}\right)$.

Conversely, assume $\left(\Delta(\lambda)-A_{\mathrm{SS}}\right)$ is not invertible for some $\lambda=\lambda_{0}$ in $\mathbb{U}$ and let $n_{0} \neq 0$ be in the corresponding null-space. Then $n$ defined by

$$
n(s)=\lambda_{0}^{s} n_{0} \text { for all } s
$$

belongs to $\ell_{2}\left(\mathbb{Z}_{2 L}\right)$ and satisfies (16) with $v \equiv 0$. Hence operator $\left(\Delta_{\mathrm{S}}-A_{\mathrm{SS}}\right)$ is not invertible on $\ell_{2}\left(\mathbb{Z}_{2 L}\right)$.

For the infinite system case, the proof of sufficiency is identical, replacing the discrete Fourier transform with a two-sided $\mathcal{Z}$-transform. For necessity, assuming that there exists $\lambda_{0}=e^{i \omega_{0}} \in \partial \mathbb{D}$ such that $\left(\Delta\left(\lambda_{0}\right)-A_{\mathrm{SS}}\right)$ is singular, we will show that $\left(\Delta_{\mathrm{S}}-A_{\mathrm{SS}}\right)$ is not bounded below on $\ell_{2}(\mathbb{Z})$ and hence not injective. Let $\xi_{0}$ be a unitary vector in the null-space of $\left(\Delta\left(\lambda_{0}\right)-A_{\mathrm{SS}}\right)$. Define the sequence of functions $\left\{u_{k}\right\}$ from $[0,2 \pi)$ to $\mathbb{R}$ by

$$
u_{k}(\omega)=\left\{\begin{array}{l}
2^{\frac{k-1}{2}} \text { if }\left|\omega-\omega_{0}\right|<\left(\frac{1}{2}\right)^{k} \\
0 \text { otherwise }
\end{array}\right.
$$

and, for each $k$, let $\hat{n}_{k} \in \ell_{2}(\partial \mathbb{D})$ be defined by $\hat{n}_{k}(\lambda)=u_{k}(\omega) \xi_{0}$ for all $\lambda=e^{i \omega}$ and $n_{k}$ be the inverse $\mathcal{Z}$-transform of $\hat{n}_{k}$, which thus belongs to $\ell_{2}(\mathbb{Z})$. We have $\left\|n_{k}\right\|_{\ell_{2}(\mathbb{Z})}=1$ for all $k$. Now, if we let $v_{k}=\left(\Delta_{\mathrm{S}}-A_{\mathrm{SS}}\right) n_{k}$ for each $k$, we get

$$
\left\|v_{k}\right\|_{\ell_{2}(\mathbb{Z})}^{2}=\left\|\hat{v}_{k}\right\|_{\ell_{2}(\partial \mathbb{D})}^{2}=2^{(k-1)} \int_{\omega-\left(\frac{1}{2}\right)^{k}}^{\omega+\left(\frac{1}{2}\right)^{k}}\left|\left(\Delta\left(e^{i \omega}\right)-A_{\mathrm{SS}}\right) n_{0}\right|^{2} d \omega .
$$

Let $\epsilon>0$. Since $\omega \mapsto\left\|\left(\Delta\left(e^{i \omega}\right)-A_{\mathrm{SS}}\right) n_{0}\right\|^{2}$ is continuous and $\left(\Delta\left(e^{i \omega_{0}}\right)-A_{\mathrm{SS}}\right) n_{0}=0$, there exists $K$ such that

$$
\left\|\left(\Delta\left(e^{i \omega}\right)-A_{\mathrm{SS}}\right) n_{0}\right\|^{2}<\epsilon^{2} \text { for all }\left|\omega-\omega_{0}\right|<\left(\frac{1}{2}\right)^{k},
$$

provided $k>K$. Hence, $\left\|v_{k}\right\|_{\ell_{2}(\mathbb{Z})}<\epsilon$ for $k>K$. Since this holds for any $\epsilon>0$, the sequence $\left\{\left\|v_{k}\right\|_{\ell_{2}(\mathbb{Z})}\right\}_{k}$ converges to zero, showing that $\left(\Delta_{\mathrm{S}}-A_{\mathrm{SS}}\right)$ is not bounded below.

Since $\mathbb{U} \subset \partial \mathbb{D}$, well-posedness of the infinite system implies well-posedness of the corresponding periodic system. For a well-posed system, one can define stability as follows.

Definition 3.2. A well-posed system is stable if, in the absence of input $(d \equiv 0)$, the weak solution $x(t) \in \ell_{2}(\mathbb{M})$ of (11a) is defined on $\mathbb{R}^{+}$and satisfies

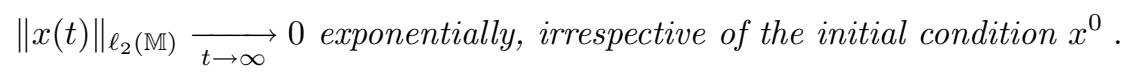


Equivalently, this means that there exist $M, \alpha>0$ such that

$$
\|\Phi(t)\|_{\ell_{2}(\mathbb{M})} \leq M e^{-\alpha t} \text { for all } t \geq 0,
$$

where the norm in the latter equation is the $\ell_{2}(\mathbb{M})$-induced norm of an operator.

It follows from the results of [1] that stability of periodic and infinite systems can be checked by looking at the corresponding Fourier-transformed systems. More precisely, if we associate the operator $\widehat{\mathbf{A}}:=\mathcal{F} \mathbf{A} \mathcal{F}^{-1}$ on $\ell_{2}(\widehat{\mathbb{M}})$ to the operator $\mathbf{A}$ on $\ell_{2}(\mathbb{M})$, where

$\widehat{\mathbb{M}}=\partial \mathbb{D}, \quad \mathcal{F}$ is the two-sided $\mathcal{Z}$-transform for an infinite system,

$\widehat{\mathbb{M}}=\mathbb{U}, \quad \mathcal{F}$ is the discrete Fourier transform for a periodic system,

then we have the following.

Proposition 3.3 (see [1]). The following hold:

(i) $\widehat{\mathbf{A}}$ is a multiplication operator; i.e., there exists a matrix-valued function $A$ such that $(\widehat{\mathbf{A}} \hat{f})(\lambda)=A(\lambda) \hat{f}(\lambda)$ for all $\hat{f} \in \ell_{2}(\widehat{\mathbb{M}})$.

(ii) The periodic and infinite system (9) is stable if and only if $A(\lambda)$ is Hurwitz for all $\lambda \in \widehat{\mathbb{M}}$.

Note that (ii) is in fact simpler than the general condition of [1] for stability, owing to the compactness of $\widehat{\mathbb{M}}$. Once again, since $\mathbb{U} \subset \partial \mathbb{D}$, stability of the infinite system implies stability of the corresponding periodic system.

It is easy to see that if a system is well-posed and stable then, for any $d \in$ $\mathcal{L}_{2}(\mathbb{M}), x$ and in turn $z$ also belong to $\mathcal{L}_{2}(\mathbb{M})$. Such a system thus has a well-defined input/output map, $T_{d z}$. It is a bounded linear map from $\mathcal{L}_{2}(\mathbb{M})$ to $\mathcal{L}_{2}(\mathbb{M})$ and its induced norm, $\left\|T_{d z}\right\|_{\mathcal{L}_{2}(\mathbb{M})}$, characterizes the performance of the system. If it is strictly less than 1 , we will say that the system is contractive.

4. Spatial reversibility and the method of images. We now turn our attention to a particular class of finite extent systems, as defined below.

DeFINITION 4.1. Given a basic building block as per (1) and a nonsingular matrix $M$, we say that the block is (spatially) $M$-reversible if there exist matrices $R \in \mathbb{R}^{m \times m}$, $P \in \mathbb{R}^{n_{\mathrm{T}} \times n_{\mathrm{T}}}$, and $U \in \mathbb{R}^{p \times p}$ such that

(i) $R^{2}=I_{m}, P^{2}=I_{n_{\mathrm{T}}}, U^{2}=I_{p}$; i.e., $R, U$, and $P$ are involutions,

(ii) $\left(\begin{array}{ccc}P & 0 & 0 \\ 0 & Q & 0 \\ 0 & 0 & U\end{array}\right)\left(\begin{array}{ccc}A_{\mathrm{TT}} & A_{\mathrm{TS}} & B_{\mathrm{T}} \\ A_{\mathrm{ST}} & A_{\mathrm{SS}} & B_{\mathrm{S}} \\ C_{\mathrm{T}} & C_{\mathrm{S}} & D\end{array}\right)=\left(\begin{array}{ccc}A_{\mathrm{TT}} & A_{\mathrm{TS}} & B_{\mathrm{T}} \\ A_{\mathrm{ST}} & A_{\mathrm{SS}} & B_{\mathrm{S}} \\ C_{\mathrm{T}} & C_{\mathrm{S}} & D\end{array}\right)\left(\begin{array}{ccc}P & 0 & 0 \\ 0 & Q & 0 \\ 0 & 0 & R\end{array}\right)$,

where $Q:=\left(\begin{array}{cc}0 & M \\ M-1 & 0\end{array}\right)$.

We will say that a finite extent, periodic, or infinite system is $M$-reversible if the basic building block is. When the finite extent system at hand has boundary conditions matrix $M$ and is $M$-reversible, we will simply say that it is reversible without referring to the matrix. Our goal in this section is to relate the properties of reversible finite extent and periodic systems. This will require several properties that we explain in turn. We start with a result that motivates our use of the notion of spatial reversibility.

First, we introduce the reflection $\Upsilon: \ell_{2}\left(\mathbb{Z}_{2 L}\right) \rightarrow \ell_{2}\left(\mathbb{Z}_{2 L}\right)$ such that $(\Upsilon x)(s)=$ 
$x(2 L+1-s)$ for all $s$. Then we consider the following spaces of reversible signals:

$$
\begin{aligned}
& \Re_{\mathrm{T}}:=\left\{x \in \ell_{2}^{n_{\mathrm{T}}}\left(\mathbb{Z}_{2 L}\right): x=P \Upsilon x\right\}, \\
& \Re_{\mathrm{S}}:=\left\{v \in \ell_{2}^{n_{\mathrm{S}}}\left(\mathbb{Z}_{2 L}\right): v=Q \Upsilon v\right\}, \\
& \Re_{d}:=\left\{d \in \ell_{2}^{m}\left(\mathbb{Z}_{2 L}\right): d=R \Upsilon d\right\}, \\
& \Re_{z}:=\left\{z \in \ell_{2}^{p}\left(\mathbb{Z}_{2 L}\right): z=U \Upsilon z\right\} .
\end{aligned}
$$

Then the fact that $Q$ and $\Upsilon$, as seen as operators on $\ell_{2}^{n_{\mathrm{S}}}\left(\mathbb{Z}_{2 L}\right)$, satisfy

$$
\Delta_{\mathrm{S}} Q=Q \Delta_{\mathrm{S}}^{-1} ; \Delta_{\mathrm{S}} \Upsilon=\Upsilon \Delta_{\mathrm{S}}^{-1}
$$

yields the following property.

Proposition 4.2. Assume periodic system (3) is $M$-reversible and well-posed. Let the initial state $x^{0}$ belong to $\Re_{\mathrm{T}}$ and disturbance $d \in \mathcal{L}_{2}\left(\mathbb{Z}_{2 L}\right)$ satisfy $d(t) \in \Re_{d}$ for all $t \geq 0$. Then the corresponding solution $(x, v, z)$ of $(8)$ on $\mathbb{R}^{+}$is spatially reversible, i.e., $x(t) \in \Re_{\mathrm{T}}, v(t) \in \Re_{\mathrm{S}}$, and $z(t) \in \Re_{z}$ for all $t \geq 0$.

The proof relies on manipulations very similar to those used later for Theorem 4.5 and we thus omit it. Physically, Proposition 4.2 means that, for the right type of inputs and initial conditions, the signals flowing to the right from the $L$ th subsystem are related to those flowing to the left from the $(L+1)$ th subsystem. Hence switching left and right is equivalent to operating $Q$ on $v, P$ on $x$, and $R$ on $z$. This property allows us to draw a parallel between spatially reversible and time-reversible dynamical systems. Recall that a nonlinear autonomous dynamical system

$$
\dot{x}=f(x)
$$

is time-reversible if there exists an involution $R$ that anticommutes with $f$. Then for every solution $x$ of the differential equation (18) we have another, i.e., $\tilde{x}: t \mapsto \tilde{x}(t)=$ $R x(-t)$. If there exists $t^{\star}$ such that $x\left(t^{\star}\right) \in F i x(R)=\{\xi: \xi=R \xi\}$, then the solution $x$ is reversible.

In both spatial and temporal cases, the key property is some kind of anticommutation of an involution with an evolution operator ((17) in the spatial case) and the result is that solutions either "come in pairs" or are reversible. It is because of this analogy that the denomination "spatially reversible" was used in our definition, although "symmetric" has sometimes been used in the literature with a somewhat similar meaning [16], [21]. This latter denomination is acceptable because (17) and Definition 4.1 essentially mean that the set of equations describing the periodic system is equivariant under the action of $\mathbb{Z}_{2}$. However, we feel that it is desirable to keep the adjective "symmetric" for systems that are invariant under the action of more general groups, as is done in [10].

The second useful result is given by the following proposition.

Proposition 4.3. A spatially reversible finite extent system is well-posed if the corresponding periodic system is well-posed.

Proof. We use a contrapositive. Assume the finite extent system is not well-posed and let $M$ be its boundary conditions matrix. We want to show that $\left(\Delta(\theta)-A_{\mathrm{SS}}\right)$ is singular for some $\theta \in \mathbb{U}$. Let $\omega=e^{i \pi / L}$ so that

$$
\mathbb{U}=\left\{1, \omega, \omega^{2}, \ldots, \omega^{(2 L-1)}\right\} .
$$

According to Proposition 3.1, there exists $x \neq 0$ such that $N x=x$, where $N$ is defined as in (14). Also, because of spatial reversibility, we have that $Q A_{\mathrm{SS}}=A_{\mathrm{SS}} Q$, 
namely,

$$
\left(\begin{array}{cc}
M A_{\mathrm{SS}_{-,-}} M^{-1} & M A_{\mathrm{SS}_{-,+}} M \\
M^{-1} A_{\mathrm{SS}_{+,-}} M^{-1} & M^{-1} A_{\mathrm{SS}_{+,+}} M
\end{array}\right)=\left(\begin{array}{cc}
A_{\mathrm{SS}_{+,+}} & A_{\mathrm{SS}_{+,-}} \\
A_{\mathrm{SS}_{-,+}} & A_{\mathrm{SS}_{-,-}}
\end{array}\right) .
$$

As a result, $\mathcal{Q}$ defined by

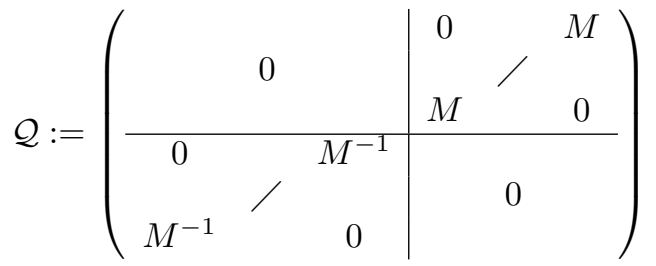

commutes with $N$ and $\mathcal{Q} x$ is also an eigenvector of $N$ with eigenvalue 1 . There are two cases as follows.

Case 1. $x=-\mathcal{Q} x$.

Since the Vandermonde matrix $V\left(\omega, \omega^{3}, \ldots, \omega^{(2 L-1)}\right)$ defined by

$$
V\left(\omega, \omega^{3}, \ldots, \omega^{(2 L-1)}\right):=\left(\begin{array}{cccc}
I_{n} & \frac{1}{\omega} I_{n} & \ldots & \left(\frac{1}{\omega}\right)^{(L-1)} I_{n} \\
I_{n} & \frac{1}{\omega^{3}} I_{n} & \ldots & \left(\frac{1}{\omega^{3}}\right)^{(L-1)} I_{n} \\
\vdots & \vdots & & \vdots \\
I_{n} & \frac{1}{\omega^{(2 L-1)}} I_{n} & \ldots & \left(\frac{1}{\omega^{(2 L-1)}}\right)^{(L-1)} I_{n}
\end{array}\right)
$$

is invertible, there exists $1 \leq k \leq L$ such that $\sum_{l=1}^{L}\left(\frac{1}{\omega^{(2 k-1)}}\right)^{(l-1)} x_{l} \neq 0$, for otherwise we would have $x_{1}=\cdots=x_{L}=0$ and, in turn, since $x=-\mathcal{Q} x$, $x=0$, a contradiction.

For this $k$, let

$$
x^{+}=\sum_{l=1}^{L}\left(\frac{1}{\omega^{(2 k-1)}}\right)^{(l-1)} x_{l} ; x^{-}=\sum_{l=1}^{L}\left(\frac{1}{\omega^{(2 k-1)}}\right)^{(l-1)} x_{(L+l)} ; X=\left(\begin{array}{l}
x^{+} \\
x^{-}
\end{array}\right) .
$$

Note that $X=-Q X \neq 0$ and

$$
\begin{aligned}
\omega^{(2 k-1)} x^{+} & =A_{\mathrm{SS}_{+},+}\left[\omega^{(2 k-1)} M x_{(L+1)}+x_{1}+\cdots+\left(\frac{1}{\omega^{(2 k-1)}}\right)^{(L-2)} x_{(L-1)}\right] \\
& +A_{\mathrm{SS}_{+,-}}\left[\omega^{(2 k-1)} M^{-1} x_{1}+x_{(L+1)}+\cdots+\left(\frac{1}{\omega^{(2 k-1)}}\right)^{(L-2)} x_{(2 L-1)}\right] \\
& =A_{\mathrm{SS}_{+},+} x^{+}+A_{\mathrm{SS}_{+},-} x^{-}
\end{aligned}
$$

since $x_{L}=-M x_{(L+1)}, x_{1}=-M x_{2 L}$, and $-\omega^{(2 k-1)}=\left(\frac{1}{\omega^{(2 k-1)}}\right)^{(L-1)}$.

Likewise, we get

$$
\left(\frac{1}{\omega^{(2 k-1)}}\right) x^{-}=A_{\mathrm{SS}_{-,+}} x^{+}+A_{\mathrm{SS}_{-,-}} x^{-} .
$$

Hence, $\left(\Delta\left(\omega^{(2 k-1)}\right)-A_{\mathrm{SS}}\right) X=0$ with $X \neq 0$. 
Case 2. $x \neq-\mathcal{Q} x$.

Define $z:=x+\mathcal{Q} x$. Since it is nonzero, it is an eigenvector of $N$ with eigenvalue 1. Also, $z=\mathcal{Q} z$. Considering $V\left(1, \omega^{2}, \ldots, \omega^{(2 L-2)}\right)$ as for Case 1, one deduces that there exists $0 \leq k \leq(L-1)$ such that $\sum_{l=1}^{L}\left(\frac{1}{\omega^{(2 k)}}\right)^{(l-1)} z_{l} \neq$ 0 .

Then, if we let

$$
z^{+}=\sum_{l=1}^{L}\left(\frac{1}{\omega^{(2 k)}}\right)^{(l-1)} z_{l} ; z^{-}=\sum_{l=1}^{L}\left(\frac{1}{\omega^{(2 k)}}\right)^{(l-1)} z_{(L+l)} ; Z=\left(\begin{array}{c}
z^{+} \\
z^{-}
\end{array}\right),
$$

we get $\left(\Delta\left(\omega^{(2 k)}\right)-A_{\mathrm{SS}}\right) Z=0$ with $Z \neq 0$, after calculations similar to those of Case 1.

Hence, in any case, the periodic system is not well-posed.

Remark 1. Note that the condition in Proposition 4.3 is sufficient but not necessary, as can be seen by considering the following case where $n=1, M=1$, and $L=2$ :

$$
A_{\mathrm{SS}}=\left(\begin{array}{ll}
3 & 2 \\
2 & 3
\end{array}\right)
$$

$A_{\mathrm{SS}}$ has an eigenvalue at 1 , which means that the periodic system is not well-posed. However, the corresponding matrix $I-N$ is

$$
I_{4}-\left(\begin{array}{cccc}
2 & 0 & 3 & 0 \\
3 & 0 & 2 & 0 \\
0 & 2 & 0 & 3 \\
0 & 3 & 0 & 2
\end{array}\right)=\left(\begin{array}{cccc}
-1 & 0 & -3 & 0 \\
-3 & 1 & -2 & 0 \\
0 & -2 & 1 & -3 \\
0 & -3 & 0 & -1
\end{array}\right)
$$

which is invertible.

In particular, this means that all analysis results pertaining to periodic systems only yield sufficient conditions for finite extent systems.

Finally, let $\mathbf{H}: \ell_{2}\left(\mathbb{Z}_{2 L}\right) \rightarrow \ell_{2}(\{1, \ldots, L\})$ be defined by

$$
(\mathbf{H} v)(s)=v(L+s) \text { for all } s=1, \ldots, L .
$$

Note that the restriction of $\mathbf{H}$ to the reversible subspaces $\Re_{\mathrm{T}}, \Re_{\mathrm{S}}, \Re_{d}$, and $\Re_{z}$ is invertible with, for example,

$$
\left(\mathbf{H}_{\mid \Re_{\mathrm{s}}}^{-1} v\right)(s)=\left\{\begin{aligned}
Q v(L+1-s) & \text { if } s \\
v(s-L) & \text { if } s=(L+1, \ldots, L, \ldots, 2 L,
\end{aligned}\right.
$$

and similar relations in the other cases.

Proposition 4.4. Assume the basic building block (1) is reversible. Then

$$
\begin{aligned}
& \mathbf{H} K=K \mathbf{H} \text { for all matrix } K, \\
& \Delta_{\mathrm{S}} \mathbf{H}_{\mid \Re_{\mathrm{S}}}^{-1}=\mathbf{H}_{\mid \Re_{\mathrm{S}}}^{-1} \Delta_{\mathrm{BC}}, \\
& A_{\mathrm{SS}} \mathbf{H}_{\mid \Re_{\mathrm{S}}}^{-1}=\mathbf{H}_{\mid \Re_{\mathrm{S}}}^{-1} A_{\mathrm{SS}},
\end{aligned}
$$

Proof. Equation (21a) is clear. Equation (21c) simply follows from the fact that $A_{\mathrm{SS}}$ and $Q$ commute. For $(21 \mathrm{~b})$, we start by showing that $\Delta_{\mathrm{BC}}=\mathbf{H} \Delta_{\mathrm{S}} \mathbf{H}_{\mid \Re_{\mathrm{S}}}^{-1}$. Let 
$w=\mathbf{H}_{\mid \Re_{\mathrm{s}}}^{-1} v, y=\Delta_{\mathrm{s}} w$ and $z=\mathbf{H} y$. Then, for all $1 \leq s \leq L$,

$$
\begin{aligned}
& z^{+}(s)=y^{+}(L+s)=w^{+}(L+s+1), \\
& z^{-}(s)=y^{-}(L+s)=w^{-}(L+s-1) .
\end{aligned}
$$

If $1 \leq s \leq L-1$, (22a) means that $z^{+}(s)=v^{+}(s+1)$. For $s=L, L+s+1=$ $2 L+1=1 \bmod 2 L$, and hence $z^{+}(L)=w^{+}(1)=M v^{-}(L)$, using (20). Likewise, for $2 \leq s \leq L, z^{-}(s)=v^{-}(s-1)$ while $z^{-}(1)=w^{-}(L)=M^{-1} v^{+}(1)$. All in all, recalling interconnection relation (7), we see that $z=\Delta_{\mathrm{BC}} v$. This shows that

$$
\Delta_{\mathrm{BC}}=\mathbf{H} \Delta_{\mathrm{S}} \mathbf{H}_{\mid \Re_{\mathrm{S}}}^{-1} .
$$

Finally, (17) implies that $\Re_{\mathrm{S}}$ is a stable subspace for operator $\Delta_{\mathrm{S}}$ (i.e., $\Delta_{\mathrm{S}} v \in \Re_{\mathrm{S}}$ if $v \in \Re_{\mathrm{S}}$ ). We can thus left-multiply (23) by $\mathbf{H}_{\mid \Re_{\mathrm{S}}}^{-1}$ to get (ii).

We are now in a position to state and prove the main theorem of this section.

THEOREM 4.5 (method of images). Let a spatially $M$-reversible finite extent system be such that the corresponding periodic system is well-posed. For an input $d \in \mathcal{L}_{2}(\{1, \ldots, L\})$ and initial state $x^{0} \in \ell_{2}(\{1, \ldots, L\})$, let $d^{P}(t):=\mathbf{H}_{\Re_{d}}^{-1} d(t)$ and $\left(x^{0}\right)^{P}:=\mathbf{H}_{\mid \Re_{\mathrm{T}}}^{-1} x^{0}$. Let $\left(x^{P}, v^{P}, z^{P}\right)$ be the spatially reversible solution of the periodic system with input $d^{P}$, initial state $\left(x^{0}\right)^{P}$. Then $(x, v, z)$ defined by $x(t):=\mathbf{H} x^{P}(t)$, $v(t):=\mathbf{H} v^{P}(t)$, and $z(t):=\mathbf{H} z(t)^{P}$ for all $t \geq 0$ is the unique solution of the finite extent system with input $d$ and initial condition $x^{0}$.

Proof. First, according to Proposition 4.3, the finite extent system is well-posed since the periodic one is. The finite system thus has a unique solution $(x, v, z)$, where $x$ is the mild solution of (11a) for $\Delta=\Delta_{\mathrm{BC}}$. Now, note that $x^{P}(t)$ satisfies

$$
x^{P}(t)=e^{t \mathbf{A}}\left(x^{0}\right)^{P}+\int_{0}^{t} e^{(t-\tau) \mathbf{A}}\left(\mathbf{B} d^{P}\right)(\tau) d \tau
$$

for $\mathbf{A}=A_{\text {TT }}+A_{\mathrm{TS}}\left(\Delta_{\mathrm{S}}-A_{\mathrm{SS}}\right)^{-1} A_{\mathrm{ST}}$ and $\mathbf{B}=B_{\mathrm{T}}+A_{\mathrm{TS}}\left(\Delta_{\mathrm{S}}-A_{\mathrm{SS}}\right)^{-1} B_{\mathrm{S}}$. Hence

$$
\left(\mathbf{H} x^{P}\right)(t)=\mathbf{H} e^{t \mathbf{A}}\left(x^{0}\right)^{P}+\mathbf{H} \int_{0}^{t} e^{(t-\tau) \mathbf{A}}\left(\mathbf{B} d^{P}\right)(\tau) d \tau .
$$

Using relation (17) and the fact that $A_{\mathrm{SS}}$ commutes with $Q$, it is easy to see that the subspace $\Re_{\mathrm{S}}$ is invariant for the mapping $\left(\Delta_{\mathrm{S}}-A_{\mathrm{SS}}\right)$. Hence, we can write

$$
\begin{aligned}
\mathbf{H}\left(\Delta_{\mathrm{S}}-A_{\mathrm{SS}}\right)_{\mid \Re_{\mathrm{S}}}^{-1} & =\left(\left(\Delta_{\mathrm{S}}-A_{\mathrm{SS}}\right)_{\mid \Re_{\mathrm{S}}} \mathbf{H}_{\mid \Re_{\mathrm{S}}}^{-1}\right)^{-1} \\
& =\left(\mathbf{H}_{\mid \Re_{\mathrm{S}}}^{-1}\left(\Delta_{\mathrm{BC}}-A_{\mathrm{SS}}\right)_{\mid \Re_{\mathrm{S}}}\right)^{-1},
\end{aligned}
$$

where we have used Proposition 4.4. This, coupled with the fact that the basic building block is reversible, yields $\mathbf{H A} x=\mathbf{A}_{\mathrm{BC}} \mathbf{H} x$ for all $x \in \Re_{\mathrm{T}}$ and $\mathbf{H B} d=\mathbf{B}_{\mathrm{BC}} \mathbf{H} d$ for all $d \in \Re_{d}$, where $\mathbf{A}_{\mathrm{BC}}=A_{\mathrm{TT}}+A_{\mathrm{TS}}\left(\Delta_{\mathrm{BC}}-A_{\mathrm{SS}}\right)^{-1} A_{\mathrm{ST}}$ and $\mathbf{B}_{\mathrm{BC}}=B_{\mathrm{T}}+A_{\mathrm{TS}}\left(\Delta_{\mathrm{BC}}-A_{\mathrm{SS}}\right)^{-1} B_{\mathrm{S}}$. In particular, this also implies that $\mathbf{H} e^{t \mathbf{A}}\left(x^{0}\right)^{P}=e^{t \mathbf{A}_{\mathrm{BC}}} \mathbf{H}\left(x^{0}\right)^{P}$, since $\left(x^{0}\right)^{P} \in \Re_{\mathrm{T}}$. All in all, plugging this back into (24) gives that

$$
\mathbf{H} x^{P}(t)=e^{t \mathbf{A}_{\mathrm{BC}}} \mathbf{H}\left(x^{0}\right)^{P}+\int_{0}^{t} e^{(t-\tau) \mathbf{A}_{\mathrm{BC}}} \mathbf{B}_{\mathrm{BC}}\left(\mathbf{H} d^{P}\right)(\tau) d \tau,
$$


i.e., that $x(t)=\mathbf{H} x^{P}(t)$ for all $t \geq 0$ since $\left(x^{0}\right)^{P}$ and $d^{P}$ are reversible by construction. Once this is known, it is clear that $v=\mathbf{H} v^{P}$ and $z=\mathbf{H} z^{P}$.

An easy but fundamental corollary is that stability and performance of a spatially reversible finite extent system are related to similar properties of the corresponding periodic and infinite system.

COROLLARY 4.6. If a spatially $M$-reversible, well-posed, periodic system is stable, then the corresponding finite extent system, with boundary conditions matrix $M$, is stable. Moreover, the input/output gains of the two systems satisfy

$$
\left\|T_{d z}\right\|_{\mathcal{L}_{2}(\{1, \ldots, L\})} \leq \sqrt{\frac{1+\bar{\sigma}(R)^{2}}{1+\underline{\sigma}(U)^{2}}}\left\|T_{d z}^{P}\right\|_{\mathcal{L}_{2}\left(\mathbb{Z}_{2 L}\right)},
$$

where $T_{d z}$ and $T_{d z}^{P}$ are the input/output map of the finite extent and periodic system, respectively. In particular, if $R$ and $U$ are unitary,

$$
\left\|T_{d z}^{P}\right\|_{\mathcal{L}_{2}\left(\mathbb{Z}_{2 L}\right)}<1 \Rightarrow\left\|T_{d z}\right\|_{\mathcal{L}_{2}(\{1, \ldots, L\})}<1 .
$$

Proof. We first prove stability. First assume that the periodic system is stable and pick an initial condition $x^{0} \in \ell_{2}(\{1, \ldots, L\})$ for the finite extent system. Let $(x, v, z)$ be the corresponding solution in the absence of an input, which is uniquely determined since we assumed well-posedness. Let $\left(x^{0}\right)^{P}$ and $x^{P}$ be defined as in Theorem 4.5. Then, Theorem 4.5 implies that, for all $t \geq 0$,

$$
\|x(t)\|_{\ell_{2}(\{1, \ldots, L\})} \leq\left\|x^{P}(t)\right\|_{\ell_{2}\left(\mathbb{Z}_{2 L}\right)} \underset{t \rightarrow \infty}{\longrightarrow} 0 \text { exponentially }
$$

since the periodic system is stable. Since this holds for any $x^{0}$, the finite extent is stable.

For performance, using the notation of Theorem 4.5, we note that

$$
\left\|d^{P}\right\|_{\mathcal{L}_{2}\left(\mathbb{Z}_{2 L}\right)}^{2} \leq\left(1+\bar{\sigma}(R)^{2}\right)\|d\|_{\mathcal{L}_{2}(\{1, \ldots, L\})}^{2} .
$$

Also $\left\|z^{P}\right\|_{\mathcal{L}_{2}\left(\mathbb{Z}_{2 L}\right)}^{2} \geq\left(1+\underline{\sigma}(U)^{2}\right)\|z\|_{\mathcal{L}_{2}(\{1, \ldots, L\})}^{2}$.

5. Examples. We now give some practical examples of spatially reversible systems and their corresponding boundary conditions.

Example 1 (two-sided platoon). The following is adapted from [19]. Consider the problem of controlling a platoon of $L$ vehicles such that each has a constant velocity $V$ and is halfway between its predecessor and successor in the line, in spite of external noise. This design requirement captures the notion of "safety" since it ensures that each vehicle is as far away as possible from its two closest neighbors. This system can be described by

$$
\begin{aligned}
& \dot{e}(t, s)=-\mathrm{v}(t, s)+\frac{1}{2}(\mathrm{v}(t, s+1)+\mathrm{v}(t, s-1)), \\
& \dot{\mathrm{v}}(t, s)=a(t, s), \\
& \dot{a}(t, s)=-a(t, s)+u(t, s)+m(t, s), \\
& z(t, s)=e(t, s) \text { for all } s=1, \ldots, L, \quad t \geq 0,
\end{aligned}
$$

where, in a frame moving with constant velocity $V, \mathrm{v}(., s), a(., s), u(., s), m(., s)$ are the velocity, acceleration, control, and external noise of the $s$ th vehicle, respectively. 
$e(., s)$ is the difference between the position of the $s$ th vehicle and the middle of its closest neighbors. This system can be put into the standard form (8) by choosing

$$
\begin{gathered}
x=\left(\begin{array}{c}
e \\
\mathrm{v} \\
a
\end{array}\right), d=\left(\begin{array}{c}
u \\
m
\end{array}\right), \quad n_{\mathrm{T}}=3, n=1, \\
A_{\mathrm{TT}}=\left(\begin{array}{ccc}
0 & -1 & 0 \\
0 & 0 & 1 \\
0 & 0 & -1
\end{array}\right), A_{\mathrm{TS}}=\left(\begin{array}{cc}
\frac{1}{2} & \frac{1}{2} \\
0 & 0 \\
0 & 0
\end{array}\right), B_{\mathrm{T}}=\left(\begin{array}{ll}
0 & 0 \\
0 & 0 \\
1 & 1
\end{array}\right), \\
A_{\mathrm{ST}}=\left(\begin{array}{ccc}
0 & 1 & 0 \\
0 & 1 & 0
\end{array}\right), A_{\mathrm{SS}}=\left(\begin{array}{ll}
0 & 0 \\
0 & 0
\end{array}\right), B_{\mathrm{S}}=\left(\begin{array}{ll}
0 & 0 \\
0 & 0
\end{array}\right), \\
C_{\mathrm{T}}=\left(\begin{array}{lll}
1 & 0 & 0
\end{array}\right), C_{\mathrm{S}}=\left(\begin{array}{ll}
0 & 0
\end{array}\right), D=\left(\begin{array}{ll}
0 & 0
\end{array}\right) .
\end{gathered}
$$

Two different sets of relevant boundary conditions can be thought of that will yield a spatially reversible system. Both involve a virtual leader, located in front of the first vehicle of the platoon, and a virtual follower located behind the $L$ th vehicle of the platoon as follows:

- The virtual leader and follower have the same velocity as the first and last vehicle of the platoon, respectively. This case can be captured by the boundary conditions matrix $M=1$ and can be used to specify that the platoon should follow the virtual leader. The corresponding finite extent system is then reversible with $P=U=R=I$.

- The virtual leader's (respectively, follower's) velocity is the opposite of the first (respectively, last) vehicle's. This boundary condition can be captured by taking $M=-1$ and corresponds to a case where the leader is reversing in front of the platoon. The corresponding finite extent system is reversible with $P=U=R=-I$.

Example 2 (heat equation). The following is the partial differential equation describing the diffusion of heat in a bar of unit length:

$$
\begin{aligned}
\frac{\partial x}{\partial t} & =\frac{\partial^{2} x}{\partial l^{2}}+d \text { for all } l \in(0,1), \quad t \geq 0, \\
x(0, l) & =x^{0}(l) \text { for all } l \in(0,1), \\
x(t, 0) & =x(t, 1)=0 \text { for all } t \geq 0 .
\end{aligned}
$$

In (27), $x(t, l) \in \mathbb{R}$ is the temperature at time $t$ and position $l \in[0,1]$, and $d(t, l)$ is a distributed heat source. The Dirichlet boundary conditions $(27 \mathrm{c})$ mean that the temperature is held constant at both ends, while initial conditions (27b) specify that the initial temperature profile is $x^{0}$.

We discretize this equation in the spatial direction using a centered finite-difference method with step $\delta_{l}$ such that $\frac{1}{\delta_{l}}=L \in \mathbb{N}$. If we write $\bar{x}(t, s)$ for the approximation of $x\left(t,\left(s-\frac{1}{2}\right) \delta_{l}\right)$ and approximate $\frac{\partial x}{\partial l}(t, l)$ to second order in $\delta_{l}$ by

$$
\frac{x\left(t, l+\frac{\delta_{l}}{2}\right)-x\left(t, l-\frac{\delta_{l}}{2}\right)}{\delta_{l}}
$$


for all $l \in[0, L]$, we get the following semidiscretized system, [3], [5]:

$$
\begin{aligned}
\frac{d \bar{x}}{d t}(t, s)= & \frac{\bar{x}(t, s+1)-2 \bar{x}(t, s)+\bar{x}(t, s-1)}{\delta_{l}^{2}} \\
& +d\left(t,\left(s-\frac{1}{2}\right) \delta_{l}\right) \text { for all } s=1, \ldots, L, \quad t \geq 0 \\
\bar{x}(0, s)= & x^{0}\left(\left(s-\frac{1}{2}\right) \delta_{l}\right)
\end{aligned}
$$

with boundary conditions

$$
\bar{x}(t, 0)=-\bar{x}(t, 1) ; \bar{x}(t, L)=-\bar{x}(t, L+1) .
$$

The latter approximate the original boundary conditions $(27 \mathrm{c})$ up to order $\delta_{l}^{2}$, which is also the order of accuracy of (28a). This system can be written as a finite extent system in standard form (8) with

$$
\begin{gathered}
x=z=\bar{x}, n_{\mathrm{T}}=1, n=1, \\
A_{\mathrm{TT}}=-\frac{2}{\delta_{l}^{2}}, A_{\mathrm{TS}}=\frac{1}{\delta_{l}^{2}}\left(\begin{array}{ll}
1 & 1
\end{array}\right), B_{\mathrm{T}}=1, \\
A_{\mathrm{ST}}=\left(\begin{array}{c}
1 \\
1
\end{array}\right), A_{\mathrm{SS}}=\left(\begin{array}{ll}
0 & 0 \\
0 & 0
\end{array}\right), B_{\mathrm{S}}=\left(\begin{array}{l}
0 \\
0
\end{array}\right), \\
C_{\mathrm{T}}=1, C_{\mathrm{S}}=\left(\begin{array}{ll}
0 & 0
\end{array}\right), D=0 .
\end{gathered}
$$

Then if we let $v^{+}(t, 1):=\bar{x}(t, 0)$ and $v^{-}(L):=\bar{x}(t, L+1)$, boundary conditions (29) can be rewritten as

$$
v^{+}(t, 1)=-w^{-}(t, 1) ; v^{-}(t, L)=-w^{+}(t, L),
$$

which corresponds to the boundary conditions matrix $M=-1$. It is then easy to see that the system is spatially reversible with $P=U=R=-I$.

6. Control synthesis for finite extent systems. We are now interested in solving the following $\mathcal{H}_{\infty}$ synthesis problem.

Problem 1. Given a finite extent system with boundary conditions (the plant), find another such system (the controller) such that the closed-loop is well-posed, stable, and contractive.

It is also desirable that the algorithm for determining a satisfactory controller be computationally tractable, irrespective of the number of subsystems in the plant. Also note that we explicitly require the controller to have the same spatial structure as the plant, as shown in Figure 2. Hence we are aiming for a distributed control strategy, as opposed to a centralized strategy (in which all the subsystems of the plant are connected to the same controller) or decentralized strategy (in which subsystems of the controller are not interconnected with each other).

As is the case for analysis, Problem 1 has counterparts for the periodic and infinite systems corresponding to the plant. Their statements are obvious and they will be referred to as the periodic synthesis problem and infinite synthesis problem, 


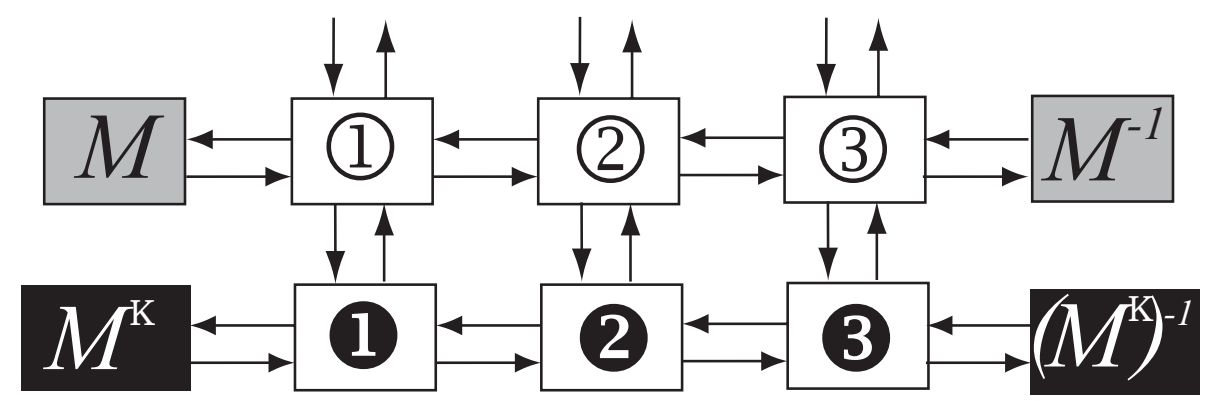

FIG. 2. Interconnection of the finite extent plant and controller for $L=3$. Note that feedback is distributed.

respectively. Both the periodic synthesis problem and the infinite synthesis problem can be efficiently solved using the methods developed in [1] and [5]. The first involves solving a family of synthesis problems, parameterized by spatial frequency while, for the second, synthesis conditions take the form of a single linear matrix inequality (LMI). However, these are only sufficient conditions. In the recent past [18], [14], Problem 1 has been tackled in the following way:

1. Solve the periodic (respectively, infinite) synthesis problem for the periodic (respectively, infinite) system corresponding to the given, finite extent, plant. This results in a periodic (respectively, infinite) controller.

2. Supplement the periodic or infinite controller's realization with some "wellchosen" boundary conditions to obtain a finite extent one solving Problem 1.

This procedure implicitly assumes that the stability and performance of the finite extent closed-loop system can be derived from the properties of the spatially invariant one and that the influence of the controller's boundary conditions can be evaluated. As already noted in [18], the last point is delicate. Bluntly stated, it is not clear what "well-chosen" boundary conditions should be in the general case.

However, we have just established that such a link between finite extent and periodic systems exists in the case of spatial reversibility. In this framework, "well-chosen" also gains a clear meaning: given a realization of the closed-loop periodic system, a well-chosen boundary conditions matrix $M^{\mathrm{K}}$ for the controller should be such that the closed-loop system is spatially reversible with some boundary conditions matrix $M^{\mathrm{C}}$. Indeed, if this is the case, Corollary 4.6 will guarantee stability and performance when the controller, which solves the periodic synthesis problem, is implemented on the finite plant, with boundary conditions $M^{\mathrm{K}}$.

In the remaining sections, we develop tools to show that $M^{\mathrm{K}}=\left(M^{*}\right)^{-1}$ is a wellchosen boundary condition for the controller if the plant, with boundary conditions matrix $M$, is spatially reversible. More precisely, we show the following.

THEOREM 6.1. Given a finite extent, spatially reversible plant, there exists a spatially reversible, finite extent controller, with $n_{\mathrm{T}}^{\mathrm{K}}=n_{\mathrm{T}}, n^{\mathrm{K}}=n$, and boundary conditions matrix $M^{\mathrm{K}}=\left(M^{*}\right)^{-1}$, that solves Problem 1 if the LMI conditions of [5] (equations (34)) are satisfied and if the plant's involutions $R$ and $U$ satisfy $R=$ $\operatorname{diag}\left(R^{u}, R^{d}\right)$ and $U=\operatorname{diag}\left(U^{y}, U^{z}\right)$ with $R^{d}=\left(R^{d}\right)^{*}=\left(R^{d}\right)^{-1}, U^{z}=\left(U^{z}\right)^{*}=$ $\left(U^{z}\right)^{-1}$.

This means that $\mathcal{H}_{\infty}$ synthesis for a spatially reversible finite extent system (with boundary conditions) can be achieved by solving a convex problem, to determine 
the controller's basic building block, and by "reading off" the controller's boundary conditions from the plant. The first step toward this synthesis result, which is also needed to make the statement of Problem 1 more rigorous, is the interconnection of reversible systems.

6.1. Interconnection of systems and spatial reversibility. The interconnection of two finite extent, periodic, or infinite systems is obtained by performing a linear fractional transformation of every pair of subsystems with the same index. This is depicted in Figure 2 in the case of finite extent systems.

More precisely, suppose we are given a plant with two sets of inputs (the exogenous disturbance $d \in \mathbb{R}^{m_{d}}$ and the control input $u \in \mathbb{R}^{m_{u}}$ ) and outputs (the performance output $z \in \mathbb{R}^{p_{z}}$ and the measured output $y \in \mathbb{R}^{p_{y}}$ ), as described by

$$
\begin{aligned}
\frac{d}{d t} x(t) & =A_{\mathrm{TT}} x(t)+A_{\mathrm{TS}} v(t)+\left(\begin{array}{cc}
B_{\mathrm{T}}^{u} & B_{\mathrm{T}}^{d}
\end{array}\right)\left(\begin{array}{c}
u(t) \\
d(t)
\end{array}\right), x(0)=x^{0} \\
\left(\Delta-A_{\mathrm{SS}}\right)[v(t)] & =A_{\mathrm{ST}} x(t)+\left(\begin{array}{cc}
B_{\mathrm{S}}^{u} & B_{\mathrm{S}}^{d}
\end{array}\right)\left(\begin{array}{c}
u(t) \\
d(t)
\end{array}\right) \\
\left(\begin{array}{c}
y(t) \\
z(t)
\end{array}\right) & =\left(\begin{array}{c}
C_{\mathrm{T}}^{y} \\
C_{\mathrm{T}}^{z}
\end{array}\right) x(t)+\left(\begin{array}{c}
C_{\mathrm{S}}^{y} \\
C_{\mathrm{S}}^{z}
\end{array}\right) v(t)+\left(\begin{array}{cc}
D^{y u} & D^{y d} \\
D^{z u} & D^{z d}
\end{array}\right)\left(\begin{array}{c}
u(t) \\
d(t)
\end{array}\right)
\end{aligned}
$$

Then its interconnection with the controller given by

$$
\begin{aligned}
\frac{d}{d t} x^{\mathrm{K}}(t) & =A_{\mathrm{TT}}^{\mathrm{K}} x^{\mathrm{K}}(t)+A_{\mathrm{TS}}^{\mathrm{K}} v^{\mathrm{K}}(t)+B_{\mathrm{T}}^{\mathrm{K}} y(t), x^{\mathrm{K}}(0)=\left(x^{\mathrm{K}}\right)^{0}, \\
\left(\Delta-A_{\mathrm{SS}}\right)\left[v^{\mathrm{K}}(t)\right] & =A_{\mathrm{ST}}^{\mathrm{K}} x^{\mathrm{K}}(t)+B_{\mathrm{S}}^{\mathrm{K}} y(t), \\
u(t) & =C_{\mathrm{T}}^{\mathrm{K}} x^{\mathrm{K}}(t)+C_{\mathrm{S}}^{\mathrm{K}} v^{\mathrm{K}}(t)+D^{\mathrm{K}} y(t) \text { for all } t \geq 0
\end{aligned}
$$

is the system obtained by eliminating $u$ and $y$ in (30) and (31). We emphasize that $\left[x^{\mathrm{K}}(t)\right](s) \in \mathbb{R}^{n_{\mathrm{T}}^{\mathrm{K}}}$ and $\left[\left(v^{+}\right)^{K}(t)\right](s),\left[\left(v^{-}\right)^{K}(t)\right](s) \in \mathbb{R}^{n^{\mathrm{K}}}$ for all $t \geq 0$, and $s$ with $n_{\mathrm{T}}^{\mathrm{K}} \neq n_{\mathrm{T}}$ and $n^{\mathrm{K}} \neq n$ a priori. The dimensions of the matrices defining operator $\Delta$ in (31b) are thus chosen accordingly.

The corresponding closed-loop system equations can be put in standard form (8) using the permutation matrix

$$
\Pi=\left(\begin{array}{cccc}
I_{n} & 0 & 0 & 0 \\
0 & 0 & I_{n^{\mathrm{K}}} & 0 \\
0 & I_{n} & 0 & 0 \\
0 & 0 & 0 & I_{n^{\mathrm{K}}}
\end{array}\right)
$$

in order to group the spatial variables properly. This means that the closed-loop system is also a finite extent, periodic, or infinite system, depending on the case. A realization of the corresponding basic building block, when $D^{y u}=0$, is given below. Note that this is not a restrictive assumption since one can always use loop-shifting 
if this situation is not at hand (see [5] and references therein):

$$
\begin{aligned}
& A_{\mathrm{TT}}^{\mathrm{C}}=\left(\begin{array}{cc}
A_{\mathrm{TT}}+B_{\mathrm{T}}^{u} D^{\mathrm{K}} C_{\mathrm{T}}^{y} & B_{\mathrm{T}}^{u} C_{\mathrm{T}}^{\mathrm{K}} \\
B_{\mathrm{T}}^{\mathrm{K}} C_{\mathrm{T}}^{y} & A_{\mathrm{TT}}^{\mathrm{K}}
\end{array}\right), \quad A_{\mathrm{TS}}^{\mathrm{C}}=\left(\begin{array}{cc}
A_{\mathrm{TS}}+B_{\mathrm{T}}^{u} D^{\mathrm{K}} C_{\mathrm{S}}^{y} & B_{\mathrm{T}}^{u} C_{\mathrm{s}}^{\mathrm{K}} \\
B_{\mathrm{T}}^{\mathrm{K}} C_{\mathrm{S}}^{y} & A_{\mathrm{TS}}^{\mathrm{K}}
\end{array}\right) \Pi, \\
& A_{\mathrm{ST}}^{\mathrm{C}}=\Pi\left(\begin{array}{cc}
A_{\mathrm{ST}}+B_{\mathrm{S}}^{u} D^{\mathrm{K}} C_{\mathrm{T}}^{y} & B_{\mathrm{S}}^{u} C_{\mathrm{T}}^{\mathrm{K}} \\
B_{\mathrm{S}}^{\mathrm{K}} C_{\mathrm{T}}^{y} & A_{\mathrm{ST}}^{\mathrm{K}}
\end{array}\right), \quad A_{\mathrm{SS}}^{\mathrm{C}}=\Pi\left(\begin{array}{cc}
A_{\mathrm{SS}}+B_{\mathrm{S}}^{u} D^{\mathrm{K}} C_{\mathrm{S}}^{y} & B_{\mathrm{S}}^{u} C_{\mathrm{S}}^{\mathrm{K}} \\
B_{\mathrm{S}}^{\mathrm{K}} C_{\mathrm{S}}^{y} & A_{\mathrm{SS}}^{\mathrm{K}}
\end{array}\right) \Pi \text {, } \\
& B_{\mathrm{T}}^{\mathrm{C}}=\left(\begin{array}{c}
B_{\mathrm{T}}^{d}+B_{\mathrm{T}}^{u} D^{\mathrm{K}} D^{y d} \\
B_{\mathrm{T}}^{\mathrm{K}} D^{y d}
\end{array}\right), \quad \quad B_{\mathrm{S}}^{\mathrm{C}}=\Pi\left(\begin{array}{c}
B_{\mathrm{S}}^{d}+B_{\mathrm{S}}^{u} D^{\mathrm{K}} D^{y d} \\
B_{\mathrm{S}}^{\mathrm{K}} D^{y d}
\end{array}\right), \\
& C_{\mathrm{T}}^{\mathrm{C}}=\left(\begin{array}{cc}
C_{\mathrm{T}}^{z}+D^{z u} D^{\mathrm{K}} C_{\mathrm{T}}^{y} & D^{z u} C_{\mathrm{T}}^{\mathrm{K}}
\end{array}\right), \quad C_{\mathrm{S}}^{\mathrm{C}}=\left(\begin{array}{cc}
C_{\mathrm{S}}^{z}+D^{z u} D^{\mathrm{K}} C_{\mathrm{S}}^{y} & D^{z u} C_{\mathrm{S}}^{\mathrm{K}}
\end{array}\right) \Pi, \\
& D^{\mathrm{C}}=D^{z d}+D^{z u} D^{\mathrm{K}} D^{y d} \text {. }
\end{aligned}
$$

Using (32), it is easy to show the following.

Proposition 6.2. Let the plant, with boundary conditions matrix $M$, and the controller, with boundary conditions matrix $M^{\mathrm{K}}$, be spatially reversible. Assume further that the involutions $R$ and $U$ for the plant and $R^{\mathrm{K}}$ and $U^{\mathrm{K}}$ for the controller satisfy $R^{\mathrm{K}}=U^{y}$ and $U^{\mathrm{K}}=R^{u}$, where $U$ and $R$ are partitioned conformably to the inputs and outputs as $R=\operatorname{diag}\left(R^{u}, R^{d}\right)$ and $U=\operatorname{diag}\left(U^{y}, U^{z}\right)$. Then their interconnection, which has $M^{\mathrm{C}}=\operatorname{diag}\left(M, M^{\mathrm{K}}\right)$ as boundary conditions matrix, is spatially reversible with $R^{\mathrm{C}}=R^{d}, P^{\mathrm{C}}=\operatorname{diag}\left(P, P^{\mathrm{K}}\right)$, and $U^{\mathrm{C}}=U^{z}$.

Proposition 6.2, combined with Corollary 4.6, already gives a way to solve Problem 1: if the periodic synthesis problem can be (tractably) solved by any means, and if the resulting controller can be shown to be $M^{\mathrm{K}}$-reversible for some matrix $M^{\mathrm{K}}$, with $R^{\mathrm{K}}=U$ and $U^{\mathrm{K}}=R$, then this is the boundary conditions matrix that should be used for the finite extent controller.

6.2. Reversible infinite controllers for reversible infinite plants. In this section we give the second element needed to establish Theorem 6.1, namely, the following.

Proposition 6.3. Consider an $M$-reversible infinite plant. Assume the involutions $R$ and $U$ for the plant are of the type indicated in Theorem 6.1. Then it is always possible to solve the infinite synthesis problem with an $\left(M^{*}\right)^{-1}$-reversible controller, provided the LMI condition of [5] (equation (34)) is satisfied. Moreover, $R^{\mathrm{K}}=U^{y}$, $U^{\mathrm{K}}=R^{u}$, and $P^{\mathrm{K}}=P^{*}$ for this controller.

It should be noted that this reversible controller is not necessarily the solution that one would obtain by directly solving (34)-(37) with a numerical solver such as those included in the LMI toolbox for MATLAB. However, if one has a solution, one can construct such an $\left(M^{*}\right)^{-1}$-reversible controller by following the steps of the proof.

Before proving Proposition 6.3, we should clarify why this implies Theorem 6.1. First, thanks to a theorem of [1] stating that the input/output gain of well-posed, stable systems over a group can be determined by a frequency-grid search, one can prove that contractiveness of the infinite system implies contractiveness of the corresponding periodic system, using arguments very similar to those of Proposition 3.3. As a result, the periodic system corresponding to the $\left(M^{*}\right)^{-1}$-reversible controller of Proposition 6.3 solves the periodic synthesis problem. The periodic closed-loop system is thus wellposed, stable, and contractive. It is also $M^{\mathrm{C}}$-reversible with $M^{\mathrm{C}}=\operatorname{diag}\left(M,\left(M^{*}\right)^{-1}\right)$ by virtue of Proposition 6.2. One can then apply the method of images to show that the corresponding finite extent closed-loop system is also well-posed and stable. Finally, since $R^{d}$ and $U^{z}$ are assumed to be unitary, Corollary 4.6 yields contractiveness of the finite extent closed-loop. 
It might seem artificial to introduce the infinite system in order to solve Problem 1 , while the method of images refers only to the periodic system. The main practical reason for using a finite controller corresponding to a reversible solution of the infinite synthesis problem is the following. Imagine the number $L$ of subsystems in the finite extent system is changed to $L^{\prime} \neq L$. Then the size of the corresponding periodic system also changes (from $2 L$ to $2 L^{\prime}$ ) and so does the corresponding group $\mathbb{U}$, which now becomes $\mathbb{U}^{\prime}$, the group of $\left(2 L^{\prime}\right)$ th root of unity. A solution of the periodic synthesis problem for $2 L$ subsystems does not necessarily solve the same problem for $2 L^{\prime}$ subsystems since well-posedness, stability, and performance of the closed-loop all depend on the group $\mathbb{U}$, and $\mathbb{U}^{\prime}$ may or may not be a subgroup of $\mathbb{U}$. Hence, if one uses a finite extent controller corresponding to a reversible solution of the periodic synthesis problem, one has to redo a synthesis if the number of subsystems in the finite extent plant changes. This is not desirable since this number is in fact irrelevant for a spatially reversible plant (only the boundary conditions matter). A reversible solution of the infinite synthesis problem, on the other hand, solves it irrespective of $L$.

Proof. The proof is by construction. For the reader's convenience and because they are used extensively in this proof, we first recall the notation and main results of $[5]$.

Given a well-posed infinite plant with basic building block (1), let

$$
H=\left(\begin{array}{cc}
I_{n} & 0 \\
0 & -I_{n}
\end{array}\right)
$$

and define the bilinear algebraic transformed system by

$$
\begin{aligned}
& \overline{A_{\mathrm{SS}}}:=H\left(A_{\mathrm{SS}}-I\right)\left(A_{\mathrm{SS}}+I\right)^{-1}, \\
& \left(\begin{array}{ll}
\overline{A_{\mathrm{ST}}} & \overline{B_{\mathrm{S}}}
\end{array}\right):=\sqrt{2} H\left(A_{\mathrm{SS}}+I\right)^{-1}\left(\begin{array}{ll}
A_{\mathrm{ST}} & B_{\mathrm{S}}
\end{array}\right), \\
& \left(\begin{array}{c}
\overline{A_{\mathrm{TS}}} \\
\overline{C_{\mathrm{S}}}
\end{array}\right):=\sqrt{2}\left(\begin{array}{c}
A_{\mathrm{TS}} \\
C_{\mathrm{S}}
\end{array}\right)\left(A_{\mathrm{SS}}+I\right)^{-1}, \\
& \left(\begin{array}{cc}
\overline{A_{\mathrm{TT}}} & \overline{B_{\mathrm{T}}} \\
\overline{C_{\mathrm{T}}} & \bar{D}
\end{array}\right):=\left(\begin{array}{cc}
A_{\mathrm{TT}} & B_{\mathrm{T}} \\
C_{\mathrm{T}} & D
\end{array}\right)-\left(\begin{array}{c}
A_{\mathrm{TS}} \\
C_{\mathrm{S}}
\end{array}\right)\left(A_{\mathrm{SS}}+I\right)^{-1}\left(\begin{array}{ll}
A_{\mathrm{ST}} & B_{\mathrm{S}}
\end{array}\right), \\
& \bar{A}^{\mathrm{G}}=\left(\begin{array}{ll}
\overline{A_{\mathrm{TT}}} & \overline{A_{\mathrm{TS}}} \\
\overline{A_{\mathrm{ST}}} & \overline{A_{\mathrm{SS}}}
\end{array}\right), \bar{B}^{\mathrm{G}}=\left(\begin{array}{c}
\overline{B_{\mathrm{T}}} \\
\overline{B_{\mathrm{S}}}
\end{array}\right), \bar{C}^{\mathrm{G}}=\left(\begin{array}{ll}
\overline{C_{\mathrm{T}}} & \overline{C_{\mathrm{S}}}
\end{array}\right), \bar{D}^{\mathrm{G}}=\bar{D} .
\end{aligned}
$$

We also define several sets of scaling matrices:

$$
\begin{aligned}
\mathcal{X}^{\mathrm{G}} & =\left\{X^{\mathrm{G}}=\operatorname{diag}\left(X_{\mathrm{T}}^{\mathrm{G}}, X_{\mathrm{S}}^{\mathrm{G}}\right), X_{\mathrm{T}}^{\mathrm{G}} \in \mathbb{R}^{n_{\mathrm{T}} \times n_{\mathrm{T}}}, X_{\mathrm{T}}^{\mathrm{G}}>0, X_{\mathrm{S}}^{\mathrm{G}} \in \mathbb{R}^{n_{\mathrm{S}} \times n_{\mathrm{s}}}, X_{\mathrm{S}}^{\mathrm{G}} \text { is symmetric }\right\}, \\
\mathcal{X}^{\mathrm{K}} & =\left\{X^{\mathrm{K}}=\operatorname{diag}\left(X_{\mathrm{T}}^{\mathrm{K}}, X_{\mathrm{S}}^{\mathrm{K}}\right), X_{\mathrm{T}}^{\mathrm{K}} \in \mathbb{R}^{n_{\mathrm{T}}^{\mathrm{K}} \times n_{\mathrm{T}}^{\mathrm{K}}}, X_{\mathrm{T}}^{\mathrm{K}}>0, X_{\mathrm{S}}^{\mathrm{K}} \in \mathbb{R}^{n_{\mathrm{S}}^{\mathrm{K}} \times n_{\mathrm{s}}^{\mathrm{K}}}, X_{\mathrm{S}}^{\mathrm{K}} \text { is symmetric }\right\}, \\
\mathcal{X}^{\mathrm{GK}} & =\left\{X=\operatorname{diag}\left(X_{\mathrm{T}}^{\mathrm{GK}}, X_{\mathrm{S}}^{\mathrm{GK}}\right), X_{\mathrm{T}}^{\mathrm{GK}} \in \mathbb{R}^{n_{\mathrm{T}} \times n_{\mathrm{T}}^{\mathrm{K}}}, X_{\mathrm{S}}^{\mathrm{GK}} \in \mathbb{R}^{n_{\mathrm{s}} \times n_{\mathrm{s}}^{\mathrm{K}}}\right\} .
\end{aligned}
$$

Then, the main synthesis result of [5] is the following.

THEOREM 6.4. Let the columns of $\mathcal{N}_{\mathrm{Y}}$ span the null space of $\left(\left({\overline{B^{u}}}^{\mathrm{G}}\right)^{*}\left(\overline{D^{z u}}\right)^{*}\right)$ and let those of $\mathcal{N}_{\mathrm{x}}$ span the null space of $\left(\left({\overline{C^{y}}}^{\mathrm{G}}\right)^{*}\left({\overline{D^{y d}}}^{\mathrm{G}}\right)^{*}\right)$, respectively. Then there exists an infinite controller such that the closed-loop is well-posed, stable, and 
contractive if there exist $X^{\mathrm{G}}$ and $Y^{\mathrm{G}}$ in $\mathcal{X}^{\mathrm{G}}$ satisfying the following LMI:

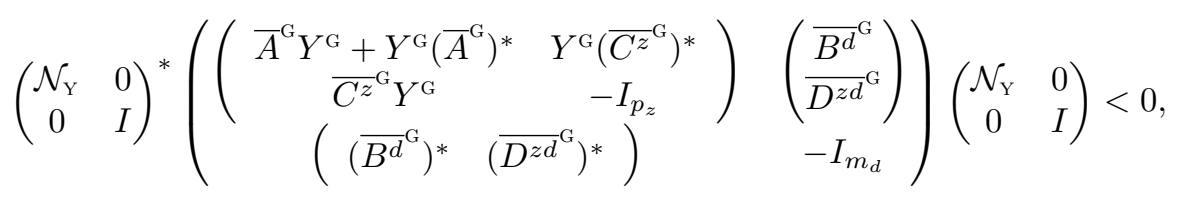

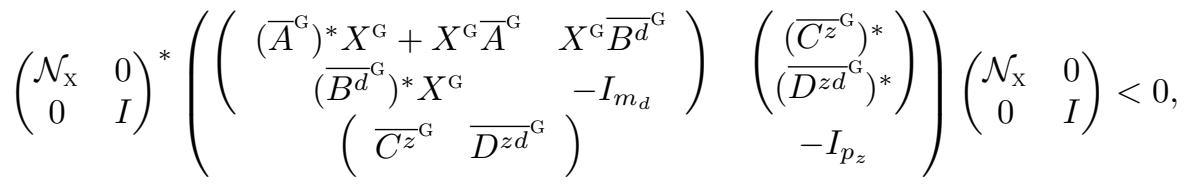

$$
\begin{aligned}
& \left(\begin{array}{cc}
X_{\mathrm{T}}^{\mathrm{G}} & I \\
I & Y_{\mathrm{T}}^{\mathrm{G}}
\end{array}\right) \geq 0 .
\end{aligned}
$$

Because (34c) is satisfied, there exist $X^{\mathrm{K}}$ and $Y^{\mathrm{K}}$ in $\mathcal{X}^{\mathrm{K}}$ and $X^{\mathrm{GK}}$ and $Y^{\mathrm{GK}}$ in $\mathcal{X}^{\mathrm{GK}}$ such that

$$
\left(\begin{array}{cc}
X^{\mathrm{G}} & X^{\mathrm{GK}} \\
\left(X^{\mathrm{GK}}\right)^{*} & X^{\mathrm{K}}
\end{array}\right)=\left(\begin{array}{cc}
Y^{\mathrm{G}} & Y^{\mathrm{GK}} \\
\left(Y^{\mathrm{GK}}\right)^{*} & Y^{\mathrm{K}}
\end{array}\right)^{-1}
$$

and $n_{\mathrm{T}}^{\mathrm{K}}=n_{\mathrm{T}}, n_{\mathrm{S}}^{\mathrm{K}}=n_{\mathrm{S}}$.

Then defining

$$
\bar{X}=\left(\begin{array}{cc}
X^{\mathrm{G}} & X^{\mathrm{GK}} \\
\left(X^{\mathrm{GK}}\right)^{*} & X^{\mathrm{K}}
\end{array}\right)
$$

we can construct a controller that solves the infinite synthesis problem in two steps as follows:

1. Solve the LMI

$$
\left(\begin{array}{ccc}
\left(\bar{A}^{\mathrm{C}}\right)^{*} \bar{X}+\overline{X A}^{\mathrm{C}} & \overline{X B}^{\mathrm{C}} & \left(\bar{C}^{\mathrm{C}}\right)^{*} \\
\left(\bar{B}^{\mathrm{C}}\right)^{*} \bar{X} & -I & \left(\bar{D}^{\mathrm{C}}\right)^{*} \\
\bar{C}^{\mathrm{C}} & \bar{D}^{\mathrm{C}} & -I
\end{array}\right)<0
$$

which is affine in the unknown

$$
\Theta:=\left(\begin{array}{ll}
\bar{A}^{\mathrm{K}} & \bar{B}^{\mathrm{K}} \\
\bar{C}^{\mathrm{K}} & \bar{D}^{\mathrm{K}}
\end{array}\right)
$$

where

$$
\left(\begin{array}{cc}
\bar{A}^{\mathrm{C}} & \bar{B}^{\mathrm{C}} \\
\bar{C}^{\mathrm{C}} & \bar{D}^{\mathrm{C}}
\end{array}\right)=\left(\begin{array}{ccc}
\bar{A}^{\mathrm{G}} & 0 & {\overline{B^{d}}}^{\mathrm{G}} \\
0 & 0 & 0 \\
\bar{C}^{z^{\mathrm{G}}} & 0 & \bar{D}^{z d}
\end{array}\right)+\left(\begin{array}{cc}
0 & {\overline{B^{u}}}^{\mathrm{G}} \\
I & 0 \\
0 & {\overline{D^{z u}}}^{\mathrm{G}}
\end{array}\right) \Theta\left(\begin{array}{ccc}
0 & I & 0 \\
\bar{C}^{\mathrm{G}} & 0 & \bar{D}^{y d}
\end{array}\right) .
$$

2. Once $\Theta$ is known, make a change of coordinates that puts ${\overline{A_{\mathrm{SS}}}}^{\mathrm{K}}$ into the form

$$
{\overline{A_{\mathrm{SS}}}}^{\mathrm{K}}=\left(\begin{array}{cc}
A^{+} & 0 \\
0 & A^{-}
\end{array}\right)
$$


where both $A^{-}$and $-\left(A^{+}\right)$are Hurwitz. This can always be achieved if ${\overline{A_{\mathrm{SS}}}}^{\mathrm{K}}$ has no eigenvalue on the imaginary axis. If this situation is not at hand, one can perturb $\overline{A_{\mathrm{SS}}}{ }^{\mathrm{K}}$ so that it holds and LMI (34) will still be satisfied. Then let

$$
H^{\mathrm{K}}=\left(\begin{array}{cc}
I_{n^{+}} & 0 \\
0 & -I_{n^{-}}
\end{array}\right)
$$

where $n^{ \pm}=\operatorname{dim}\left(A^{ \pm}\right)$, and invert the bilinear algebraic transformation by

$$
\begin{aligned}
& A_{\mathrm{SS}}^{\mathrm{K}}:=\left(H^{\mathrm{K}}-{\overline{A_{\mathrm{SS}}}}^{\mathrm{K}}\right)^{-1}\left(H^{\mathrm{K}}+{\overline{A_{\mathrm{SS}}}}^{\mathrm{K}}\right), \\
& \left(\begin{array}{ll}
A_{\mathrm{ST}}^{\mathrm{K}} & B_{\mathrm{S}}^{\mathrm{K}}
\end{array}\right):=\sqrt{2}\left(H^{\mathrm{K}}-{\overline{A_{\mathrm{SS}}}}^{\mathrm{K}}\right)^{-1}\left({\overline{A_{\mathrm{ST}}}}^{\mathrm{K}} \quad{\overline{B_{\mathrm{S}}}}^{\mathrm{K}}\right), \\
& \left(\begin{array}{c}
A_{\mathrm{TS}}^{\mathrm{K}} \\
C_{\mathrm{S}}^{\mathrm{K}}
\end{array}\right):=\sqrt{2}\left(\begin{array}{c}
\overline{A_{\mathrm{TS}}} \\
{\overline{C_{\mathrm{S}}}}^{\mathrm{K}}
\end{array}\right)\left(H^{\mathrm{K}}-{\overline{A_{\mathrm{SS}}}}^{\mathrm{K}}\right)^{-1} H^{\mathrm{K}} \\
& \left(\begin{array}{ll}
A_{\mathrm{TT}}^{\mathrm{K}} & B_{\mathrm{T}}^{\mathrm{K}} \\
C_{\mathrm{T}}^{\mathrm{K}} & D^{\mathrm{K}}
\end{array}\right):=\left(\begin{array}{ll}
\overline{A_{\mathrm{TT}}} & \overline{B_{\mathrm{T}}}{ }^{\mathrm{K}} \\
{\overline{C_{\mathrm{T}}}}^{\mathrm{K}} & \overline{D^{\mathrm{K}}}
\end{array}\right)+\left(\begin{array}{c}
\overline{{A_{\mathrm{TS}}}^{\mathrm{K}}} \\
\overline{{C_{\mathrm{S}}}^{\mathrm{K}}}
\end{array}\right)\left(H^{\mathrm{K}}-{\overline{A_{\mathrm{SS}}}}^{\mathrm{K}}\right)^{-1}\left({\overline{A_{\mathrm{ST}}}}^{\mathrm{K}}{\overline{B_{\mathrm{S}}}}^{\mathrm{K}}\right),
\end{aligned}
$$

to find the building block of an infinite controller solving the infinite synthesis problem.

Now assume that the plant at hand is $M$-reversible for some boundary conditions matrix $M$ with $R^{d}=\left(R^{d}\right)^{*}=\left(R^{d}\right)^{-1}$ and $U^{z}=\left(U^{z}\right)^{*}=\left(U^{z}\right)^{-1}$. Because of spatial reversibility and since $Q H=-H Q$, it is easy to see that

$$
V \bar{A}^{\mathrm{G}} W=\bar{A}^{\mathrm{G}}, \bar{C}^{\mathrm{G}} W=U \bar{C}^{\mathrm{G}}, V \bar{B}^{\mathrm{G}}=\bar{B}^{\mathrm{G}} R, U \bar{D}^{\mathrm{G}}=\bar{D}^{\mathrm{G}} R,
$$

where $V:=\left(\begin{array}{cc}P & 0 \\ 0 & -Q\end{array}\right)$ and $W:=\left(\begin{array}{cc}P & 0 \\ 0 & Q\end{array}\right)$.

Let $X^{\mathrm{G}}, Y^{\mathrm{G}}$ solve $(34 \mathrm{a})-(34 \mathrm{~b})$ for $\mathcal{N}_{\mathrm{X}}$ and $\mathcal{N}_{\mathrm{Y}}$. Then, pre- and postmultiplying (34a) by

$$
\left(\begin{array}{cc}
I & 0 \\
0 & \left(R^{d}\right)^{*}
\end{array}\right) \text { and }\left(\begin{array}{cc}
I & 0 \\
0 & R^{d}
\end{array}\right)
$$

(34b) by

$$
\left(\begin{array}{cc}
I & 0 \\
0 & U^{z}
\end{array}\right) \text { and }\left(\begin{array}{cc}
I & 0 \\
0 & \left(U^{z}\right)^{*}
\end{array}\right)
$$

and using (39), we get that $V^{*} X^{\mathrm{G}} W, W Y^{\mathrm{G}} V^{*} \in \mathcal{X}^{\mathrm{G}}$ also satisfy (34a)-(34b) but with $\tilde{\mathcal{N}}_{\mathrm{X}}=\left(\begin{array}{cc}W & 0 \\ 0 & R^{d}\end{array}\right) \mathcal{N}_{\mathrm{X}}$ and $\tilde{\mathcal{N}}_{\mathrm{Y}}=\left(\begin{array}{cc}V^{*} & 0 \\ 0 & \left(U^{z}\right)^{*}\end{array}\right) \mathcal{N}_{\mathrm{Y}}$, the columns of which also satisfy the assumptions of Theorem 6.4.

Now an important point is that $X^{\mathrm{G}}$ and $Y^{\mathrm{G}}$ also satisfy $(34 \mathrm{a})-(34 \mathrm{~b})$ for $\tilde{\mathcal{N}}_{\mathrm{X}}$ and $\tilde{\mathcal{N}}_{\mathrm{Y}}$. In fact, the matrices do not matter as long as their columns span the appropriate null-spaces. (The reason why it is so can be easily understood if one follows the usual procedure for formulating $\mathcal{H}_{\infty}$ synthesis as a convex problem, as presented, e.g., in Chapter 7 of [7]. See Lemma 7.2 in particular.)

Hence averaging the two sets of LMIs, we see that

$$
\tilde{X}^{\mathrm{G}}=\frac{1}{2}\left(X^{\mathrm{G}}+V^{*} X^{\mathrm{G}} W\right) \text { and } \tilde{Y}^{\mathrm{G}}=\frac{1}{2}\left(Y^{\mathrm{G}}+W Y^{\mathrm{G}} V^{*}\right)
$$


solve (34) for $\tilde{\mathcal{N}}_{\mathrm{X}}$ and $\tilde{\mathcal{N}}_{\mathrm{Y}}$. Note that

$$
Q^{*} \tilde{X}^{\mathrm{G}} Q=-\tilde{X}^{\mathrm{G}} \text { and } Q \tilde{Y}^{\mathrm{G}} Q^{*}=-\tilde{Y}^{\mathrm{G}} .
$$

In order to solve (35), one can choose a full-rank controller by picking

$$
X_{\mathrm{S}}^{\mathrm{GK}}=\left(I-\tilde{X}_{\mathrm{S}}^{\mathrm{G}} \tilde{Y}_{\mathrm{S}}^{\mathrm{G}}\right), X_{\mathrm{S}}^{\mathrm{K}}=-\left(X_{\mathrm{S}}^{\mathrm{GK}}\right)^{*} \tilde{Y}_{\mathrm{S}}^{\mathrm{G}}, Y_{\mathrm{S}}^{\mathrm{GK}}=I .
$$

Then easy algebra yields

$$
Q^{*} X_{\mathrm{S}}^{\mathrm{GK}} Q^{*}=X_{\mathrm{S}}^{\mathrm{GK}}, Q X_{\mathrm{S}}^{\mathrm{K}} Q^{*}=-X_{\mathrm{S}}^{\mathrm{K}},
$$

and, in turn, that $\bar{X}$ as per (36) satisfies

$$
\left(\begin{array}{cc}
W^{*} & 0 \\
0 & V
\end{array}\right) \bar{X}\left(\begin{array}{cc}
V & 0 \\
0 & W^{*}
\end{array}\right)=\bar{X}=\bar{X}^{*}=\left(\begin{array}{cc}
V^{*} & 0 \\
0 & W
\end{array}\right) \bar{X}\left(\begin{array}{cc}
W & 0 \\
0 & V^{*}
\end{array}\right) .
$$

Using this scaling and pre- and postmultiplying (37) by

$$
\left(\begin{array}{cccc}
W^{*} & 0 & 0 & 0 \\
0 & V & 0 & 0 \\
0 & 0 & \left(R^{d}\right)^{*} & 0 \\
0 & 0 & 0 & U^{z}
\end{array}\right) \text { and }\left(\begin{array}{cccc}
W & 0 & 0 & 0 \\
0 & V^{*} & 0 & 0 \\
0 & 0 & R^{d} & 0 \\
0 & 0 & 0 & \left(U^{z}\right)^{*}
\end{array}\right) \text {, }
$$

we see that if $\Theta$ is a solution, so is $\hat{\Theta}:=\left(\begin{array}{cc}W^{*} & 0 \\ 0 & R^{u}\end{array}\right) \Theta\left(\begin{array}{cc}V^{*} & 0 \\ 0 & U^{y}\end{array}\right)$ because of (39). Hence, $\tilde{\Theta}:=\frac{1}{2}(\Theta+\hat{\Theta})$ also satisfies LMI (37). Note that $\left(\begin{array}{cc}W^{*} & 0 \\ 0 & R^{u}\end{array}\right) \tilde{\Theta}\left(\begin{array}{cc}V^{*} & 0 \\ 0 & U^{y}\end{array}\right)=\tilde{\Theta}$, which means that the corresponding controller is such that

$$
\begin{aligned}
& \left(\begin{array}{c}
P^{*} \overline{A_{\mathrm{TS}}}{ }^{\mathrm{K}} \\
R^{u}{\overline{C_{\mathrm{S}}}}^{\mathrm{K}}
\end{array}\right) Q^{*}=-\left(\begin{array}{c}
\overline{A_{\mathrm{TS}}}{ }^{\mathrm{K}} \\
\overline{C_{\mathrm{S}}}
\end{array}\right),
\end{aligned}
$$

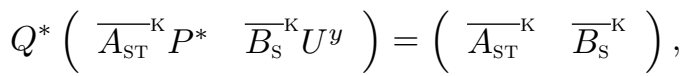

$$
\begin{aligned}
& Q^{*}{\overline{A_{\mathrm{SS}}}}^{\mathrm{K}} Q^{*}=-{\overline{A_{\mathrm{SS}}}}^{\mathrm{K}} \text {, } \\
& P^{*}{\overline{A_{\mathrm{TT}}}}^{\mathrm{K}} P^{*}={\overline{A_{\mathrm{TT}}}}^{\mathrm{K}}, P^{*}{\overline{B_{\mathrm{T}}}}^{\mathrm{K}}={\overline{B_{\mathrm{T}}}}^{\mathrm{K}} U^{y}, \\
& {\overline{C_{\mathrm{T}}}}^{\mathrm{K}} P^{*}=R^{u}{\overline{C_{\mathrm{T}}}}^{\mathrm{K}}, R^{u} \bar{D}^{\mathrm{K}} U^{y}=\bar{D}^{\mathrm{K}} .
\end{aligned}
$$

This last relation implies that the spectrum of ${\overline{A_{\mathrm{SS}}}}^{\mathrm{K}}$ is symmetric with respect to the origin. Thus, if it does not contain any point on the imaginary axis, we will have $n^{+}=\operatorname{dim}\left(A^{+}\right)=\operatorname{dim}\left(A^{-}\right)=n^{-}$. Also, because we picked a full-rank controller, we have $n_{\mathrm{S}}^{\mathrm{K}}=n_{\mathrm{S}}$ and thus $n^{ \pm}=n$ and $H^{\mathrm{K}}=H$.

Now, plugging (40) into (38), we get

$$
\begin{aligned}
& \left(\begin{array}{c}
P^{*} A_{\mathrm{TS}}^{\mathrm{K}} \\
R^{u} C_{\mathrm{S}}^{\mathrm{K}}
\end{array}\right) Q^{*}=-\left(\begin{array}{c}
A_{\mathrm{TS}}^{\mathrm{K}} \\
C_{\mathrm{S}}^{\mathrm{K}}
\end{array}\right), \\
& Q^{*}\left(\begin{array}{ll}
A_{\mathrm{ST}}^{\mathrm{K}} P^{*} & B_{\mathrm{S}}^{\mathrm{K}} U^{y}
\end{array}\right)=-\left(\begin{array}{ll}
A_{\mathrm{ST}}^{\mathrm{K}} & B_{\mathrm{S}}^{\mathrm{K}}
\end{array}\right), \\
& Q^{*} A_{\mathrm{SS}}^{\mathrm{K}} Q^{*}=A_{\mathrm{SS}}^{\mathrm{K}}, \\
& P^{*} A_{\mathrm{TT}}^{\mathrm{K}} P^{*}=A_{\mathrm{TT}}^{\mathrm{K}}, P^{*} B_{\mathrm{T}}^{\mathrm{K}}=B_{\mathrm{T}}^{\mathrm{K}} U^{y}, \\
& C_{\mathrm{T}}^{\mathrm{K}} P^{*}=C_{\mathrm{T}}^{\mathrm{K}}, R^{u} D^{\mathrm{K}} U^{y}=D^{\mathrm{K}} .
\end{aligned}
$$

Finally, perform a state transformation on $v^{\mathrm{K}}$,

$$
v^{\mathrm{K}} \rightarrow H v^{\mathrm{K}},
$$

to yield an $\left(M^{*}\right)^{-1}$-reversible controller with $R^{\mathrm{K}}=U^{y}, U^{\mathrm{K}}=R^{u}$, and $P^{\mathrm{K}}=P^{*}$ 
7. Some generalizations. In this section, we explain how some assumptions can be relaxed and our results extended to more general, spatially multidimensional, reversible systems.

7.1. The case where $\boldsymbol{R}^{d}$ and $\boldsymbol{U}^{\boldsymbol{z}}$ are not unitary. Although Theorem 6.1 treats the case where both $R^{d}$ and $U^{z}$ are unitary, it is possible to handle cases where $\left(R^{d}\right)^{*} \neq\left(R^{d}\right)^{-1}$ or $\left(U^{z}\right)^{*} \neq\left(U^{z}\right)^{-1}$ as well. There are two cases as follows:

- $\bar{\sigma}\left(R^{d}\right) \leq \underline{\sigma}\left(U^{z}\right)$ :

If one replaces the $-I_{m_{d}}$ and $-I_{p_{z}}$ blocks in (34a)-(34b) by $-\left(R^{d}\right)^{*} R^{d}$ and $-U^{z}\left(U^{z}\right)^{*}$, respectively, i.e., if one starts with an infinite controller such that

$$
\left\|T_{d z}\right\|_{\mathcal{L}_{2}(\mathbb{Z})}<\frac{\bar{\sigma}\left(R^{d}\right)}{\underline{\sigma}\left(U^{z}\right)}
$$

then following all the steps of the proof will yield an $\left(M^{*}\right)^{-1}$-reversible infinite controller such that the closed-loop system is well-posed, stable, and contractive. In turn, the corresponding periodic closed-loop system will also be contractive and, since

$$
\sqrt{\frac{1+\underline{\sigma}\left(U^{z}\right)^{2}}{1+\bar{\sigma}\left(R^{d}\right)^{2}}} \geq 1
$$

Corollary 4.6 implies that the finite extent closed-loop system is also contractive.

- $\bar{\sigma}\left(R^{d}\right)>\underline{\sigma}\left(U^{z}\right)$ :

In this case, it is possible to construct an $\left(M^{*}\right)^{-1}$-rever sible infinite controller that guarantees contractiveness of the closed-loop system if the LMIs (34a)(34b) have a solution. The corresponding periodic closed-loop will also be contractive but the finite extent one need not be. We have only the upperbound

$$
\left\|T_{d z}\right\|_{\mathcal{L}(\{1, \ldots, L\})}<\sqrt{\frac{1+\underline{\sigma}\left(U^{z}\right)^{2}}{1+\bar{\sigma}\left(R^{d}\right)^{2}}} .
$$

7.2. Multiple spatial dimensions. It is straightforward to extend our present results to cases where the subsystems are distributed on a multidimensional grid instead of a line. The basic building block then has two interconnection inputs $\left(v_{i}^{+}\right.$, $\left.v_{i}^{-} \in \mathbb{R}^{n_{i}}\right)$ and outputs $\left(w_{i}^{+}, w_{i}^{-} \in \mathbb{R}^{n_{i}}\right)$ per spatial dimension. The index $s$ used to describe the finite extent interconnection now belongs to a cartesian product set of the form

$$
\mathbb{M}=\mathbb{Z}_{l_{1}} \times \cdots \times \mathbb{Z}_{l_{k}} \times\left\{1, \ldots, L_{1}\right\} \times \cdots \times\left\{1, \ldots, L_{d}\right\}
$$

where $k \geq 0, d>0$ and there is a boundary conditions matrix $M_{i}, 1 \leq i \leq d$ associated with every nonperiodic spatial dimension. Likewise, the corresponding periodic and infinite systems are indexed over the set

$$
\mathbb{M}=\mathbb{Z}_{l_{1}} \times \cdots \times \mathbb{Z}_{l_{k}} \times \mathbb{Z}_{2 L_{1}} \times \cdots \times \mathbb{Z}_{2 L_{d}} \text { and } \mathbb{M}=\mathbb{Z}_{l_{1}} \times \cdots \times \mathbb{Z}_{l_{k}} \times \mathbb{Z}^{d},
$$

respectively. Examples of such spatially multidimensional finite extent systems are given in Figure 3 for $k=1, d=1$ and $k=0, d=2$. 


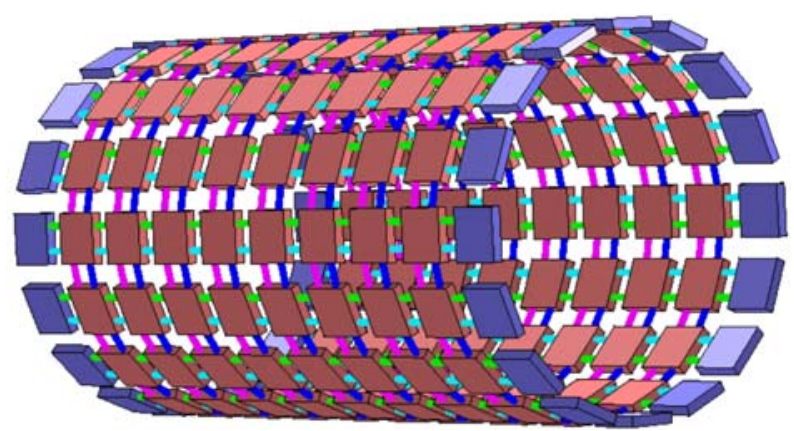

(a)

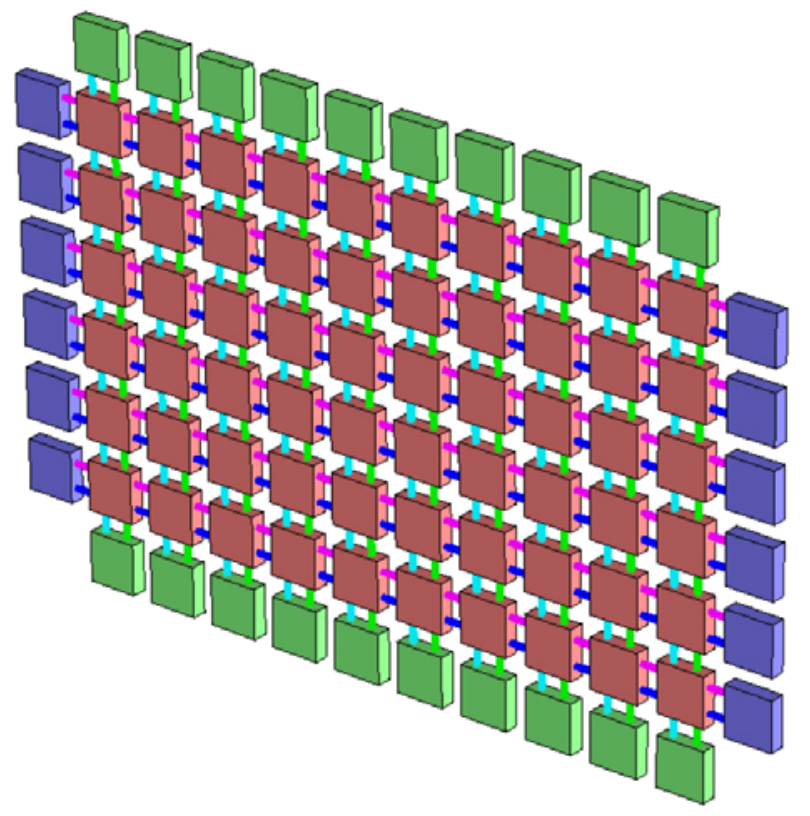

(b)

FIG. 3. Examples of spatially multidimensional interconnections. The inputs and outputs have been omitted for clarity. (a) All boundary conditions matrices are equal to $M_{1}$. (b) All boundary conditions matrices for lines (respectively, columns) are equal to $M_{1}$ (respectively, $M_{2}$ ).

These spatially multidimensional systems can be represented by (9) if we let

$$
v(t):=\left(v_{1}^{+}(t), v_{1}^{-}(t), \ldots, v_{k+d}^{+}(t), v_{k+d}^{-}(t)\right) \in \ell_{2}^{n_{\mathrm{s}}}(\mathbb{M})
$$

for a suitable $n_{\mathrm{S}}$ and replace $\Delta_{\mathrm{BC}}$ and $\Delta_{\mathrm{S}}$ by multidimensional spatial operators that capture all $k+d$ dimensions.

For example, if $k=1, d=1$, and $\mathbf{S}_{j}$ is the shift operator in the $j$ th spatial dimension $(1 \leq j \leq k+d), \Delta_{\mathrm{S}}$ should then be taken to be the structured operator

$$
\Delta_{\mathrm{S}}=\operatorname{diag}\left(\mathbf{S}_{1} I_{n_{1}}, \mathbf{S}_{1}^{-1} I_{n_{1}}, \mathbf{S}_{2} I_{n_{2}}, \mathbf{S}_{2}^{-1} I_{n_{2}}\right) .
$$

Spatial reversibility can then be defined as in the spatially monodimensional case, the only difference being that the basic building block's realization must now commute with several different matrices, one for each of the $d$ spatial dimensions. 
Definition 7.1. For $1 \leq i \leq d$, let $\mathcal{M}_{i}:=\left(\begin{array}{cc}0 & M_{i} \\ M_{i}^{-1} & 0\end{array}\right)$ and

$$
Q_{i}:=\operatorname{diag}\left(I_{n_{1}}, \ldots, I_{n_{k}}, I_{n_{k+1}}, \ldots, \mathcal{M}_{i}, \ldots, I_{n_{k+d}}\right) .
$$

We say that the basic building block and, in turn, the interconnections are spatially reversible if, for all $i$, there exist matrices $R_{i} \in \mathbb{R}^{m \times m}, P_{i} \in \mathbb{R}^{n_{\mathrm{T}} \times n_{\mathrm{T}}}$, and $U_{i} \in \mathbb{R}^{p \times p}$ such that

(i) $R_{i}, U_{i}$, and $P_{i}$ are involutions;

(ii) $R_{i} R_{j}=R_{j} R_{i}, P_{i} P_{j}=P_{j} P_{i}$, and $U_{i} U_{j}=U_{j} U_{i}$ for all $1 \leq i, j \leq d$;

(iii)

$$
\begin{aligned}
& \left(\begin{array}{ccc}
P_{i} & 0 & 0 \\
0 & Q_{i} & 0 \\
0 & 0 & U_{i}
\end{array}\right)\left(\begin{array}{ccc}
A_{\mathrm{TT}} & A_{\mathrm{TS}} & B_{\mathrm{T}} \\
A_{\mathrm{ST}} & A_{\mathrm{SS}} & B_{\mathrm{S}} \\
C_{\mathrm{T}} & C_{\mathrm{S}} & D
\end{array}\right) \\
& =\left(\begin{array}{ccc}
A_{\mathrm{TT}} & A_{\mathrm{TS}} & B_{\mathrm{T}} \\
A_{\mathrm{ST}} & A_{\mathrm{SS}} & B_{\mathrm{S}} \\
C_{\mathrm{T}} & C_{\mathrm{S}} & D
\end{array}\right)\left(\begin{array}{ccc}
P_{i} & 0 & 0 \\
0 & Q_{i} & 0 \\
0 & 0 & R_{i}
\end{array}\right) .
\end{aligned}
$$

Condition (ii) is essential for the application of the method of images: it ensures that one can extend the finite extent system by reflection in the $d$ spatial dimensions to yield a periodic one.

One can then proceed to analyze and perform distributed control synthesis for multidimensional spatially reversible systems. All proofs are similar to the spatially monodimensional case but require more intensive notational bookkeeping. The two most important results, corresponding to Corollary 4.6 and Theorem 6.1, are given below for the case where $R_{i}$ and $U_{i}$ are unitary for all $1 \leq i \leq d$.

THEOREM 7.2. If a spatially multidimensional, reversible, well-posed, periodic system is stable, then the corresponding finite extent system is stable. Moreover, if $R_{i}$ and $U_{i}$ are unitary for all $1 \leq i \leq d$, the input/output gains of the two systems satisfy

$\left\|T_{d z}^{P}\right\|_{\mathcal{L}_{2}\left(\mathbb{Z}_{l_{1}} \times \cdots \times \mathbb{Z}_{l_{k}} \times \mathbb{Z}_{2 L_{1}} \times \cdots \times \mathbb{Z}_{2 L_{d}}\right)}<1 \Rightarrow\left\|T_{d z}\right\|_{\mathcal{L}_{2}\left(\mathbb{Z}_{l_{1}} \times \cdots \times \mathbb{Z}_{l_{k}} \times\left\{1, \ldots, L_{1}\right\} \times \cdots \times\left\{1, \ldots, L_{d}\right\}\right)}<1$.

THEOREM 7.3. Given a finite extent, spatially multidimensional reversible plant, there exists a spatially reversible, finite extent controller, with $n_{\mathrm{T}}^{\mathrm{K}}=n_{\mathrm{T}}, n^{\mathrm{K}}=n$, and boundary conditions matrix $M_{i}^{\mathrm{K}}=\left(M_{i}^{*}\right)^{-1}$, for each $1 \leq i \leq d$, that solves Problem 1 if

(i) the LMI conditions of [5] for the spatially multidimensional case are satisfied;

(ii) the plant's involutions $R_{i}$ and $U_{i}$ satisfy $R_{i}=\operatorname{diag}\left(R_{i}^{u}, R_{i}^{d}\right), U_{i}=\operatorname{diag}\left(U_{i}^{y}, U_{i}^{z}\right)$ with $R_{i}^{d}$ and $U_{i}^{z}$ unitary for all $1 \leq i \leq d$.

The conditions of item (i) are (34a), (34b), and (34c), supplemented by a fourth LMI (equation (92) in [5]) needed to guarantee that the matrix ${\overline{A_{\mathrm{SS}}}}^{\mathrm{K}}$ yields an implementable controller. This LMI is always trivially satisfied in the spatially monodimensional case or when ${\overline{A_{\mathrm{SS}}}}^{\mathrm{K}}$ is block diagonal. We refer to [5] for more details.

The spatially multidimensional reversible controller is constructed iteratively as follows: First, starting with any satisfactory controller and following steps that are identical to those of the proof of Proposition 6.3, we get controller number 1 such that

$$
\left(\begin{array}{ccc}
P_{1} & 0 & 0 \\
0 & Q_{1}^{*} & 0 \\
0 & 0 & U_{1}
\end{array}\right)\left(\begin{array}{ccc}
A_{\mathrm{TT}}^{\mathrm{K}} 1 & A_{\mathrm{TS} 1}^{\mathrm{K}} & B_{\mathrm{T}}^{\mathrm{K}} \\
A_{\mathrm{ST} 1}^{\mathrm{K}} & A_{\mathrm{SS} 1}^{\mathrm{K}} & B_{\mathrm{S} 1}^{\mathrm{K}} \\
C_{\mathrm{T} 1}^{\mathrm{K}} & C_{\mathrm{S} 1}^{\mathrm{K}} & D_{1}^{\mathrm{K}}
\end{array}\right)=\left(\begin{array}{ccc}
A_{\mathrm{TT}}^{\mathrm{K}} & A_{\mathrm{TS}}^{\mathrm{K}} & B_{\mathrm{T}}^{\mathrm{K}} \\
A_{\mathrm{ST}}^{\mathrm{K}} & A_{\mathrm{SS}}^{\mathrm{K}} & B_{\mathrm{S}}^{\mathrm{K}} \\
C_{\mathrm{T} 1}^{\mathrm{K}} & C_{\mathrm{S} 1}^{\mathrm{K}} & D_{1}^{\mathrm{K}}
\end{array}\right)\left(\begin{array}{ccc}
P_{1} & 0 & 0 \\
0 & Q_{1}^{*} & 0 \\
0 & 0 & R_{1}
\end{array}\right) .
$$


Then starting with controller number 1 and proceeding similarly, we get controller number 2 such that

$$
\left(\begin{array}{ccc}
P_{2} & 0 & 0 \\
0 & Q_{2}^{*} & 0 \\
0 & 0 & U_{2}
\end{array}\right)\left(\begin{array}{ccc}
A_{\mathrm{TT}}^{\mathrm{K}} 2 & A_{\mathrm{TS}}^{\mathrm{K}} & B_{\mathrm{T} 2}^{\mathrm{K}} \\
A_{\mathrm{ST}}{ }^{\mathrm{K}} & A_{\mathrm{SS}} 2 & B_{\mathrm{S} 2}^{\mathrm{K}} \\
C_{\mathrm{T} 2}^{\mathrm{K}} & C_{\mathrm{S} 2}^{\mathrm{K}} & D_{2}^{\mathrm{K}}
\end{array}\right)=\left(\begin{array}{ccc}
A_{\mathrm{TT}}^{\mathrm{K}} & A_{\mathrm{TS}}^{\mathrm{K}} & B_{\mathrm{T}}^{\mathrm{K}} \\
A_{\mathrm{ST}}^{\mathrm{K}} & A_{\mathrm{SS}}{ }_{\mathrm{K}}^{\mathrm{K}} & B_{\mathrm{S} 2}^{\mathrm{K}} \\
C_{\mathrm{T} 2}^{\mathrm{K}} & C_{\mathrm{S} 2}^{\mathrm{K}} & D_{2}^{\mathrm{K}}
\end{array}\right)\left(\begin{array}{ccc}
P_{2} & 0 & 0 \\
0 & Q_{2}^{*} & 0 \\
0 & 0 & R_{2}
\end{array}\right) .
$$

The final controller, obtained after $d$ such iterations, is spatially reversible because for $1 \leq i, j \leq d, P_{i}$ and $P_{j}, Q_{i}$ and $Q_{j}, R_{i}$ and $R_{j}$, and $U_{i}$ and $U_{j}$ commute.

8. Conclusion. We have shown that a finite extent spatially reversible system is closely related to its periodic and infinite extensions and have demonstrated that synthesis for such systems can be performed with existing tools developed in the context of spatial invariance.

The synthesis LMI conditions that we use - which were already sufficient only for well-posedness, stability, and contractiveness of an infinite system [5] - are even more conservative for finite extent systems since well-posedness of the spatially invariant systems is not necessary for well-posedness of the finite extent system. However, when these LMI are feasible, the obtained controller guarantees stability and performance irrespective of the number of subsystems in the finite extent interconnection, with obvious consequences for system reconfiguration and fault tolerance. The boundary conditions matrix of the plant is the only relevant parameter.

\section{REFERENCES}

[1] B. Bamieh, F. Paganini, and M. Dahleh, Distributed control of spatially invariant systems, IEEE Trans. Automat. Control, 47 (2002), pp. 1091-1118.

[2] H. T. Banks, R. C. Smith, And Y. Wang, Smart Material Structures Modeling, Estimation and Control, John Wiley and Sons, New York, 1996.

[3] R. W. Brockett and J. L. Willems, Discretized partial differential equations: Examples of control systems defined on modules, Automatica, 10 (1974), pp. 507-515.

[4] R. F. Curtain And H. J. Zwart, An Introduction to Infinite-Dimensional Linear System Theory, Texts in Applied Mathematics 21, Springer-Verlag, Berlin, New York, 1995.

[5] R. D'Andrea And G. E. Dullerud, Distributed control of spatially interconnected systems, IEEE Trans. Automat. Control, 48 (2003), pp. 1478-1495.

[6] B. Dionne, M. Golubitsky, And I. Stewart, Coupled cells with internal symmetry I and II, Nonlinearity, 9 (1996), pp. 559-599.

[7] G. E. Dullerud and F. Paganini, A Course in Robust Control Theory, Texts in Applied Mathematics 36, Springer-Verlag, Berlin, New York, 1999.

[8] G. E. Dullerud, R. D'Andrea, And S. G. Lall, Control of spatially varying distributed systems, in Proceedings of the 37th IEEE Conference on Decision and Control, Tampa, FL, 1998, pp. 1889-1893.

[9] L. C. Evans, Partial Differential Equations, Graduate Studies in Mathematics 19, AMS, Providence, RI, 1998

[10] F. Fagnani And J. C. Willems, Representations of symmetric linear dynamical systems, SIAM J. Control Optim., 31 (1993), pp. 1267-1293.

[11] V. Kapila, A. G. Sparks, J. Buffington, and Q. Yan, Spacecraft formation flying: Dynamics and control, in Proceedings of the American Control Conference, San Diego, CA, 1999, pp. 4137-4141.

[12] P. P. Khargonekar, K. Poolla, and A. Tannenbaum, Robust control of linear timeinvariant plants using periodic compensation, IEEE Trans. Automat. Control, 27 (1982), pp. 627-638.

[13] D. Laughlin, M. Morari, and R. D. Braatz, Robust performance of cross-directional basisweight control in paper machines, Automatica, 29 (1993), pp. 1395-1410.

[14] S. Mijanovic, G. E. Stewart, G. A. Dumont, and M. S. Davies, $\mathcal{H}_{\infty}$ robustification of a paper machine cross-directional control system, in Proceedings of the American Control Conference, 2001, Arlington, VA, pp. 2203-2209. 
[15] F. Paganini and B. Bamien, Decentralization properties of optimal distributed controllers, in Proceedings of the 37th IEEE Conference on Decision and Control, Tampa, FL, 1998, pp. $1877-1882$.

[16] L. QIU, On the robustness of symmetric systems, Systems Control Lett., 27 (1996), pp. 187-190.

[17] E. D. Sontag, Linear systems over commutative rings: A survey, Ricerche Automat., 7 (1976), pp. $1-34$.

[18] G. E. Stewart, Analysis and Design of Boundary Conditions for a Spatially Distributed Control System, Tech. report, Honeywell Industrial Control, 2001.

[19] D. Swaroop AND J. K. Hedrick, Constant spacing strategies for platooning in automated highway systems, J. Dynam. Systems, Measurement Control, 121 (1999), pp. 462-470.

[20] M. E. TaYlor, Partial Differential Equations I: Basic Theory, Springer-Verlag, Berlin, New York, 1996.

[21] G. YAng, J. L. WAng, And Y. C. Soh, Decentralized fixed modes of symmetric systems, in Proceedings of the American Control Conference, Arlington, VA, 2001, pp. 3134-3135.

[22] J. D. Wolfe, D. F. ChichKa, And J. L. Speyer, Decentralized Controllers for Unmanned Aerial Vehicle Formation Flight, Tech. report 96-3833, American Institute of Aeronautics and Astronautics, Reston, VA, 1996. 